\title{
MULTICOMPONENT FLOW IN A POROUS MEDIUM. ADSORPTION AND SORET EFFECT PHENOMENA: LOCAL STUDY AND UPSCALING PROCESS
}

\author{
Serge Blancher ${ }^{1}$, René CrefF ${ }^{1}$, Gérard Gagneux ${ }^{2}$, Bruno Lacabanne $^{2}$, \\ FranÇOIS MONTEL ${ }^{3}$ AND DAVID TRUJILlo ${ }^{2}$
}

\begin{abstract}
Our aim here is to study the thermal diffusion phenomenon in a forced convective flow. A system of nonlinear parabolic equations governs the evolution of the mass fractions in multicomponent mixtures. Some existence and uniqueness results are given under suitable conditions on state functions. Then, we present a numerical scheme based on a "mixed finite element" method adapted to a finite volume scheme, of which we give numerical analysis. In a last part, we apply an homogenization technique to the studied equations in order to obtain an efficient modelling of Soret effect and adsorption in a porous medium at a macroscopic scale.
\end{abstract}

Résumé. On étudie un système d'équations paraboliques non linéaires modélisant l'évolution des fractions massiques d'un fluide multiconstituant dans un écoulement convectif forcé sous l'influence d'un gradient thermique. Des résultats d'existence et d'unicité sont donnés sous des hypothèses relatives aux fonctions d'état des équations. On propose ensuite une méthode numérique de type "éléments finis mixtes" aboutissant à un schéma "volumes finis" dont on effectue l'analyse et la présentation des premiers résultats. Nous appliquons enfin une technique d'homogénéisation aux équations étudiées dans le but d'obtenir une modélisation macroscopique fidèle des phénomènes d'adsorption et d'effet Soret en milieu poreux.

Mathematics Subject Classification. 35K55, 35K60, 65N30.

Received: November 14, 2000. Revised: February 7, 2001.

\section{INTRODUCTION}

We present the mathematical study of a model describing the coupling between adsorption and second order terms such as molecular diffusion and thermodiffusion occurring in a multicomponent fluid inside a capillary column. The results of this study have a very wide application field which extends from the modelization of a chromatographic column to an oil layer. In the example of a chromatographic column, when sorption effects

Keywords and phrases. System of nonlinear parabolic equations, Soret effect, separation by thermal diffusion, mixed finite element, finite volume scheme, homogenization, two scale convergence.

1 Laboratoire de Transferts thermiques, Université de Pau et des Pays de l'Adour, Technopole Hélioparc, av. du Pdt Angot, 64000 Pau, France.

${ }^{2}$ Laboratoire de Mathématiques Appliquées, ERS 2055, I.P.R.A., Université de Pau et des Pays de l'Adour, 64000 Pau, France. e-mail: bruno.lacabanne@univ-pau.fr

3 Elf E.P., Centre Scientifique et Technique Jean Feger, av. Larribau, 64000 Pau, France. 
are only considered, the system is then described by first order hyperbolic equations ( $c f$. particularly [17]), but in practice, it is necessary to take into account diffusive transport and dispersive phenomena. In the case of an oil layer, thermodiffusion and adsorption effects which are often neglected in production, are essential when one has to describe the initial distribution of hydrocarbons at the field scale (cf. [20]). The mass conservation equation for the $k^{t h}$ specie, in the absence of chemical reaction, is then given by,

$$
\partial_{t}\left(N_{k}+\varphi_{k}(\mathbf{N})\right)+\operatorname{div}\left(\vec{J}_{k}\right)=0, \quad(1 \leq k \leq n)
$$

where $\vec{J}_{k}$ is the mass flux associated with the weight fraction $N_{k}$ of component $k$ and $\varphi_{k}($.$) is a function of the$ vectorial variable, called "adsorption isotherm", describing the quantity of matter present in the porous matrix of the column. This function allows then to reduce the unknowns number since we are only interested in the species present in the mobile phase, the fluid. The aspect of adsorption that we favor here is the thermodynamic equilibrium, i.e. a state where component segregation between the two phases (the stationary and the mobile one) is established and does not depend on the initial conditions. The expression of the functions family $\left(\varphi_{k}\right)_{k}$ is obtained using statistical thermodynamic and does not take into account any kinetic factor (the delay term $\varphi_{k}$ does not depend directly on time). Separation results thus from the coupling between forced motion and retention, i.e. between thermodynamic and hydrodynamic phenomena. The total matter flux, sum of the diffusive (Fick's law), convective and thermodiffusive fluxes is finally given by

$$
\overrightarrow{J_{k}}=-\sum_{j=1}^{n} D_{k j} \vec{\nabla} N_{j}+N_{k} \mathbf{U}-D_{\theta}^{k} N_{k}\left(\sum_{j=1}^{n} N_{j}-N_{k}\right) \vec{\nabla} \theta .
$$

One can recognize in this formulation the diffusive term (which includes the expressions of the "cross"-diffusion terms) to which we add two first order terms (in comparison with the unknowns), the forced convective term (along a known velocity field $\mathbf{U}$ ) and the thermodiffusive one or "Soret effect" term (which is mainly characterized by the positive coefficients $\left(S_{t}^{i}\right)$ and the term " $\vec{\nabla} \theta$ ", representative of the internal thermal gradient). The goal is to prove that the considered problem is a well posed one (in the Hadamard 's sense): given a "regular velocity" profile, we consider the following equations:

$$
\left\{\begin{array}{l}
\mathbf{U} \cdot \vec{\nabla} \theta-\kappa \Delta \theta=0 \\
\partial_{t}\left[N_{i}+k^{i}\left(N_{1}, \ldots, N_{n}\right)\right]+\mathbf{U} \cdot \vec{\nabla} N_{i}-\sum_{j=1}^{n} D_{i j} \Delta N_{j}-\operatorname{div}\left(S_{t}^{i} N_{i}\left(\sum_{j=1}^{n} N_{j}-N_{i}\right) \vec{\nabla} \theta\right)=0 \\
(1 \leq i \leq n), \text { in a roll } Q=] 0, T\left[\times \Omega, \Omega \subset \mathbb{R}^{3}\right.
\end{array}\right.
$$

associated to initial and boundary conditions in accordance with experiments, where $\Omega$ is a bounded connex open part of $\mathbb{R}^{3}$, with a Lipschitz border $\Gamma$, divided according to the rule

$$
\Gamma=\partial \Omega=\Gamma_{e} \cup \Gamma_{l} \cup \Gamma_{s} \cup \partial \Gamma_{l}, \mathcal{L}^{2}-\operatorname{meas}\left(\Gamma_{e} \cup \Gamma_{s}\right)>0,
$$

where $\Gamma_{e}\left(\right.$ resp. $\left.\Gamma_{s}\right)$ denotes the inlet (resp. the outlet) section of the fluid, and $\Gamma_{l}$ the watertight and adiabatic wall of the considered domain. We add to these equations some boundary conditions of non homogeneous Dirichlet and homogeneous Neumann conditions and an initial condition, for the complete formulation of the problem on the parabolic border. In order to enlight the mathematical structure of the model and to keep control of the analysis in the case of possible correction of state laws, we consider a more general formulation. 
We introduce the following system of partial derivative equations with the boundary conditions

$$
\left(E_{i}\right)\left\{\begin{array}{l}
\partial_{t} N_{i}+\sum_{j=1}^{n} \frac{\partial k^{i}(\mathbf{N})}{\partial x_{j}} \partial_{t} N_{j}-\sum_{j=1}^{n} D_{i j} \Delta N_{j}+\vec{\nabla}\left(\mu\left(N_{i}\right)\right) \cdot \mathbf{U}+\operatorname{div}\left(\sigma^{i}(\mathbf{N}) \vec{\nabla} \theta\right)=0 \\
\text { on }] 0, T[\times \Omega, \text { under the following boundary conditions: } \\
N_{i}(0, .)=N_{i}^{0}, \quad N_{i}=N_{i}^{\text {bord }} \text { on } \Gamma_{e} \cup \Gamma_{s}, \quad \frac{\partial N_{i}}{\partial n}=0 \text { on } \Gamma_{l}, \mathbf{U} \cdot \vec{n}=0 \text { and } \frac{\partial \theta}{\partial n}=0 \text { on } \Gamma_{l} \\
\mathbf{U} \text { and } \vec{\nabla} \theta \text { being given steady and regular vector fields, such as: } \\
\vec{\nabla} \theta \in\left(L^{\infty}(\Omega)\right)^{3}, \quad \Delta \theta \in L^{2}(\Omega), \quad \operatorname{div}(\mathbf{U})=0, \quad 1 \leq i \leq n,
\end{array}\right.
$$

where $\mu$ denotes a Lipschitz function from $\mathbb{R}$ to $\mathbb{R}$ such as $\mu(0)=0$ and $\left(\sigma^{i}\right)$ denotes a functions family from $\mathbb{R}^{n}$ to $\mathbb{R}$ such as $\sigma^{i}\left(N_{1}, \ldots, N_{i-1}, 0, N_{i+1}, \ldots, N_{n}\right)=0$, extended in such a way that $\sigma^{i}(\mathbf{N})=0$ on $\left\{\mathbf{N} \in \mathbb{R}^{n}, N_{i} \leq 0\right\}$.

We propose here the following framework:

First, we prove the existence of a solution of the problem, by establishing a priori estimate from a linearized problem. Then we use a fixed point method (Schauder Tychonov), usually used for the treatment of quasi-linear parabolic conservative laws in porous media (cf. Gagneux and Madaune-Tort [14]).

Secondly, we prove that this solution verifies physical criterions in particular cases, that are useful for the experiments such as a space dimension equal to 1 (case of the capillary tube).

Then we set up a uniqueness and a continuity dependence result for some ad hoc topologies of the solution against the initial state and some parameters of the convective transport by considering an auxiliary problem, following the transposition method introduced by Antontsev and Domanskï [4] (cf. on this point too Gagneux [13] or Holmgren [25], pp. 66-68).

Having proved the well-posedness of this problem, we present the numerical analysis of a computation scheme in it's mixed formulation.

A last part is dedicated to the application of homogenization techniques to the upscaling of equations describing thermodiffusion and effects in porous media. Two extreme situations are studied: the irreversible and full reversible cases.

We introduce in a classic way the functional Hilbert space

$$
\mathcal{V}=\left\{v \in H^{1}(\Omega), \operatorname{trace}(v)=0 \text { on } \Gamma_{e} \cup \Gamma_{s}\right\}
$$

The steady dynamical and thermal profiles are supposed to be given a priori, with the regularity given above. We consider an incompressible laminar flow (we know that thermal diffusion and viscosity are altered by turbulence).

\section{Remarks.}

In the following part, bold letters will denote vectorial lengths.

The considered problem gives a non homogeneous Dirichlet condition on $\left(\Gamma_{e} \cup \Gamma_{s}\right)$ which is treated with the help of the introduction of a variational vectorial inequation ( $c f .[7]$, p. 143, [12] or [18]) relative to the closed convex part $K$ of $\left(H^{1}(\Omega)\right)^{n}$, defined by

$$
K=\prod_{i=1}^{n} K_{i}, \quad \text { where } K_{i}=\left\{v \in H^{1}(\Omega),\left.\quad v\right|_{\Gamma_{e} \cup \Gamma_{s}}=N_{i}^{b}\right\} \text { and } \mathcal{V}=K_{i}-K_{i}
$$

One has to take care that each of $K_{i}$ is nonempty, which is supposed here, i.e. precisely, each function $N_{i}^{b}$ is sufficiently regular $\left(N_{i}^{\text {bord }} \in H^{\frac{1}{2}}\left(\Gamma_{e} \cup \Gamma_{s}\right)\right.$, defined as the space of the restrictions to $\left(\Gamma_{e} \cup \Gamma_{s}\right)$ of the functions in $H^{\frac{1}{2}}(\Gamma)$ (cf. on this point, [8], Vol. 4, Chap. VII, $\left.\S 2\right)$ ). 
In order to write scalar equations under a vectorial form, we introduce the following notations: $\mathbf{N}=\left(\begin{array}{l}N_{1} \\ \ldots \\ N_{n}\end{array}\right)$ the weight fractions vector, and $\mathbf{V}=\left(\begin{array}{c}V_{1} \\ \ldots \\ V_{n}\end{array}\right)$ the "test" functions vector, taken a priori in $(\mathcal{V})^{n}$. We introduce the following matrixes:

$$
\tilde{\mathbf{K}}\left(x_{1}, \ldots, x_{n}\right)=\left(\begin{array}{lll}
K_{11}(\mathbf{x}) & \ldots & K_{1 n}(\mathbf{x}) \\
\ldots & \ldots & \ldots \\
K_{n 1}(\mathbf{x}) & \ldots & K_{n n}(\mathbf{x})
\end{array}\right) \text {, where } K_{i j}(\mathbf{x})=\delta_{i j}+\frac{\partial k^{i}}{\partial x_{j}}(\mathbf{x}) \text {, for }(1 \leq i, j \leq n) \text { and for }
$$

$\mathbf{x} \in\left(\mathbb{R}^{+}\right)^{n}$, then for $\mathbf{x} \in \mathbb{R}^{n}$, after ad hoc extension (where $\delta_{i j}$ denotes the Kronecker's symbol). The diffusive tensor is defined by $D=\left[D_{i j}\right]_{1 \leq i, j \leq n}, D_{i j} \in \mathbb{R}$. We will give assumptions easy to verify in practice that will be sufficient for the mathematical analysis of the model.

By multiplying each equation $\left(E_{i}\right)$ by the correspondent component $V_{i}$ in $\mathcal{V}$ and by integrating on $\Omega$, we obtain, with the help of Green's formula, the vectorial variational problem, in order to define a strong solution: Find $\mathbf{N} \in\left(L^{\infty}\left(0, T ; H^{1}(\Omega)\right) \cap H^{1}(Q)\right)^{n}, N(t,.) \in K$ a.e. in t, such as, a.e. $\left.t \in\right] 0, T\left[, \forall \mathbf{V} \in\left(L^{2}(0, T ; \mathcal{V})\right)^{n}\right.$,

$$
(\mathbf{P})\left\{\begin{array}{c}
\left(\tilde{\mathbf{K}}(\mathbf{N}) \partial_{t} \mathbf{N}, \mathbf{V}\right)+d(\mathbf{N}, \mathbf{V})+U(\mathbf{N}, \mathbf{V})+\sigma(\mathbf{N}, \theta, \mathbf{V})=0 \\
\mathbf{N}(0, .)=\mathbf{N}^{0}
\end{array}\right.
$$

where we will have first defined $d, U$, $\sigma$ et (., .) in the following way, by using the sum convention on the repeated suffix:

$$
\begin{aligned}
& d(\mathbf{N}, \mathbf{V})=D_{i j} \int_{\Omega} \vec{\nabla} N_{j} \cdot \vec{\nabla} V_{i} \mathrm{~d} x, \quad U(\mathbf{N}, \mathbf{V})=-\int_{\Omega} \mu\left(N_{i}\right) \mathbf{U} \cdot \vec{\nabla} V_{i} \mathrm{~d} x \\
& \sigma(\mathbf{N}, \theta, \mathbf{V})=-\int_{\Omega} \sigma^{i}(\mathbf{N}) \vec{\nabla} \theta \cdot \vec{\nabla} V_{i} \mathrm{~d} x, \quad\left(\tilde{\mathbf{K}}(\mathbf{N}) \partial_{t} \mathbf{N}, \mathbf{V}\right)=\int_{\Omega} K_{i j}(\mathbf{N}) \partial_{t} N_{j} V_{i} \mathrm{~d} x .
\end{aligned}
$$

We are induced to study the existence of a strong solution of a coupled system of nonlinear parabolic evolutive equations, with transport and nonlinear thermal convection terms and with mixed boundary conditions. One has to prove that this formulation is a well posed one, in the Hadamard sense.

Assumption 1. The matrix $\tilde{\mathbf{K}}(\mathbf{x})$ and the diffusive tensor have a dominant diagonal, i.e. they verify the "pseudo-ellipticity" conditions, independent of $\mathbf{x}$ :

$$
(\mathcal{H})\left\{\begin{array}{l}
\exists \alpha>0, \forall \mathbf{x} \in \mathbb{R}^{n}, \forall \boldsymbol{\zeta} \in \mathbb{R}^{n}, \quad(\tilde{\mathbf{K}}(\mathbf{x}) \boldsymbol{\zeta}, \boldsymbol{\zeta}) \geq \alpha\|\boldsymbol{\zeta}\|^{2}, \\
\exists d>0, \forall \mathbf{x} \in \mathbb{R}^{n}, \forall \boldsymbol{\zeta} \in \mathbb{R}^{n}, \quad(\mathbf{D}(\mathbf{x}) \boldsymbol{\zeta}, \boldsymbol{\zeta}) \geq d\|\boldsymbol{\zeta}\|^{2} .
\end{array}\right.
$$

Assumption 2. We consider moreover that the diffusive tensor is a symmetrical one i.e.

$$
\forall(i, j) \in\{1, . ., n\}^{2} \quad D_{i j}=D_{j i} .
$$

This last assumption, which is in agreement with physical experiments, is commonly adopted in the literature (Bia and Combarnous [6]; Duvaut and Lions [12]).

First, for technical arguments, we treat the problem for the following regularity conditions:

$$
\mu \in C^{1}(\mathbb{R}) \cap W^{1,+\infty}(\mathbb{R}), \mu(0)=0, \quad \sigma^{i} \in C^{1}\left(\mathbb{R}^{n}\right) \cap W^{1,+\infty}\left(\mathbb{R}^{n}\right) .
$$


Then, considering a density argument, we will be able to free ourselves from the differentiability assumption, and to keep the only Lipschitz feature of the state function (which is closer to the reality).

\section{An existence Result}

In a first time and for more writing conveniences, we define the following functions family:

$$
\mu_{i}^{k} \equiv \frac{\partial \sigma^{i}}{\partial x_{k}}
$$

Considering a vector $\mathbf{f}=\left(f_{1}, \ldots, f_{n}\right)^{\top}$ taken in $\left(H^{1}(Q)\right)^{n}$ and the problem $\left(P_{\text {lin }}(\mathbf{f})\right)$ (with the Einstein's convention)

$$
\left\{\begin{array}{l}
\text { Find } \mathbf{N} \in\left(L^{\infty}\left(0, T ; H^{1}(\Omega)\right)\right)^{n}, \text { such as } \frac{\partial N}{\partial t} \in\left(L^{2}(Q)\right)^{n}, \\
\text { solution of the paralinearized Cauchy's problem in } Q=] 0, T[\times \Omega \\
\partial_{t} N_{i}+\frac{\partial k^{i}(\mathbf{f})}{\partial x_{j}} \partial_{t} N_{j}-D_{i j} \Delta N_{j}+\mu^{\prime}\left(f_{i}\right) \mathbf{U} \cdot \vec{\nabla} N_{i}+\sigma^{i}(\mathbf{f}) \Delta \theta+\left(\mu_{i}^{k}(\mathbf{f}) \frac{\partial N_{k}}{\partial x_{j}}\right) \frac{\partial \theta}{\partial x_{j}}=0 \\
N_{i}(0, .)=N_{i}^{0}, \\
\text { associated to boundary conditions : } \\
N_{i}=N_{i}^{\text {bord }} \text { on } \Gamma_{e} \cup \Gamma_{s}, \frac{\partial N_{i}}{\partial n}=0 \text { on } \Gamma_{l}, \mathbf{U} \cdot \vec{n}=0 \text { and } \frac{\partial \theta}{\partial n}=0 \text { on } \Gamma_{l} \quad(1 \leq i \leq n)
\end{array}\right.
$$

which owns a unique solution, with the help of the Lions theorem, applied to vectorial equations $([18]$ Chap. 3; [19])) and under the previous assumptions, it is clear that the solution $\mathbf{N}$ belongs to $\left(L^{\infty}\left(0, T ; H^{1}(\Omega)\right) \cap H^{1}(Q)\right)^{n}$.

\subsection{A priori estimates in an appropriated functional frame}

Proposition 1.1. The analysis of the paralinearized problem $\left(P_{\text {lin }}(\mathbf{f})\right)$ allows to establish the following a priori estimates:

$$
\begin{aligned}
& \exists C_{1}>0, \text { such that, } \forall \mathbf{f} \in\left(H^{1}(Q)\right)^{n},\|\mathbf{N}\|_{\left(L^{\infty}\left(0, T ; \mathrm{H}^{1}(\Omega)\right)\right)^{n}} \leq C_{1} \\
& \exists C_{2}>0, \text { such that, } \forall \mathbf{f} \in\left(H^{1}(Q)\right)^{n},\left\|\partial_{t} \mathbf{N}\right\|_{L^{2}\left(0, T ; L^{2}(\Omega)^{n}\right)} \leq C_{2}
\end{aligned}
$$

these constants depending only on $\mathbf{N}^{0}, d, \alpha$, on the various Lipschitz constants of the state functions and on $\|\mathbf{U}\|_{\left(L^{\infty}(Q)\right)^{n}}, T,|\vec{\nabla} \theta|_{\left(L^{\infty}(Q)\right)^{n}},|\Delta \theta|_{L^{2}(Q)}$.

Proof. By multiplying each equation by $\partial_{t} N_{i}$ (that can be justified by replacing $\partial_{t} N_{i}$ by a differential quotient) and by summing on $i$, we obtain the inequality, after having integrated successively on $\Omega$ and on $[0, \tau]$ where $\tau \in[0, T]:$

$$
\begin{aligned}
\iint_{\Omega \times[0, T]} K_{i j}(\mathbf{f}) \partial_{t} N_{j} \partial_{t} N_{i} \mathrm{~d} x \mathrm{~d} t-\iint_{\Omega \times[0, T]} D_{i j} \Delta N_{j} \partial_{t} N_{i} \mathrm{~d} x \mathrm{~d} t \leq & \\
\iint_{\Omega \times[0, T]}\left|\mu^{\prime} r\left(f_{i}\right) \vec{\nabla} N_{i} \cdot \mathbf{U} \partial_{t} N_{i}\right| \mathrm{d} x \mathrm{~d} t+\iint_{\Omega \times[0, T]}\left|\sigma^{i}(\mathbf{f}) \partial_{t} N_{i} \Delta \theta\right| \mathrm{d} x \mathrm{~d} t & +\iint_{\Omega \times[0, T]}\left|\mu_{i}^{k}(\mathbf{f}) \frac{\partial N_{k}}{\partial x_{j}} \partial_{t} N_{i} \frac{\partial \theta}{\partial x_{j}}\right| \mathrm{d} x \mathrm{~d} t .
\end{aligned}
$$


We denote by $\|\mathbf{N}\|_{n}^{2}=\sum_{i}\left(\int_{\Omega}\left|\vec{\nabla} N_{i}\right|^{2} \mathrm{~d} x\right)$, and observing that the expression

$$
\left[\|\mathbf{N}\|_{n}^{2}+\sum_{i}\left(\int_{\Gamma_{e} \cup \Gamma_{s}}\left(\operatorname{trace}\left(N_{i}\right)\right)^{2} \mathrm{~d} \sigma\right)\right]^{\frac{1}{2}}
$$

defines on $H^{1}(\Omega)^{n}$ an equivalent norm to the usual one; it comes

$$
\int_{0}^{\tau}\left(\tilde{\mathbf{K}}(x) \partial_{t} \mathbf{N}, \partial_{t} \mathbf{N}\right) \mathrm{d} t \geq \alpha \int_{0}^{\tau}\left|\partial_{t} \mathbf{N}\right|_{n}^{2} \mathrm{~d} t
$$

and, by considering that the diffusive tensor is symmetrical and permanent, we can write

$$
\iint_{\Omega \times[0, T]} D_{i j} \vec{\nabla} N_{j} \cdot \vec{\nabla}\left(\partial_{t} N_{i}\right) \mathrm{d} x \mathrm{~d} t \geq \frac{d}{2}\|\mathbf{N}(\tau)\|_{n}^{2}-\sum_{i, j} \frac{D_{i j}}{2}\|\mathbf{N}(0)\|_{n}^{2} .
$$

Moreover, thanks to the Young's inequality, and for a positive constant $\varepsilon$, we have,

$$
\begin{aligned}
\int_{\Omega}\left|\mu^{\prime}\left(f_{i}\right) \vec{\nabla} N_{i} \cdot \mathbf{U} \partial_{t} N_{i}\right| \mathrm{d} x & \leq C(\varepsilon)\|\mathbf{N}\|_{n}^{2}+\frac{\varepsilon}{3}\left|\partial_{t} \mathbf{N}\right|_{n}^{2} \\
\int_{\Omega}\left|\sigma^{i}(\mathbf{f}) \partial_{t} N_{i} \Delta \theta\right| \mathrm{d} x & \leq C^{\prime}(\varepsilon)|\Delta \theta|_{L^{2}(\Omega)}^{2}+\frac{\varepsilon}{3}\left|\partial_{t} \mathbf{N}\right|_{n}^{2} \\
\int_{\Omega}\left|\mu_{i}^{k}(\mathbf{f}) \frac{\partial N_{k}}{\partial x_{j}} \partial_{t} N_{i} \frac{\partial \theta}{\partial x_{j}}\right| \mathrm{d} x & \leq C^{\prime \prime}(\varepsilon)\|\mathbf{N}\|_{n}^{2}+\frac{\varepsilon}{3}\left|\partial_{t} \mathbf{N}\right|_{n}^{2}
\end{aligned}
$$

where $C^{\prime \prime}(\varepsilon)$ depends on $\left|\mu_{k}^{i}\right|_{L^{\infty}(\Omega)},|\vec{\nabla} \theta|_{L^{\infty}(Q)}$, and $C(\varepsilon)$ depends on $\|\vec{\nabla} \mu\|_{\left(L^{\infty}(\Omega)\right)^{n}},\|\mathbf{U}\|_{\left(L^{\infty}(\Omega)\right)^{n}}$. Thus we obtain the inequality, for all $\tau$ in $[0, T]$,

$$
\alpha \int_{0}^{\tau}\left|\partial_{t} \mathbf{N}\right|_{n}^{2} \mathrm{~d} t+\frac{d}{2}\|\mathbf{N}(\tau)\|_{n}^{2} \leq C_{1}\left(\mathbf{N}^{0}, \theta\right)+\varepsilon \int_{0}^{\tau}\left|\partial_{t} \mathbf{N}\right|_{n}^{2} \mathrm{~d} t+C(\varepsilon) \int_{0}^{\tau}\|\mathbf{N}(s)\|_{n}^{2} \mathrm{~d} s
$$

where $C(\varepsilon)=C\left(\varepsilon,\left|\mu_{k}^{i}\right|_{L^{\infty}(\Omega)},|\vec{\nabla} \theta|_{L^{\infty}(Q)},\|\vec{\nabla} \mu\|_{\left(L^{\infty}(\Omega)\right)^{n}},\|\|_{\left(L^{\infty}(\Omega)\right)^{n}}\right)$.

Then we choose $\varepsilon$ small enough and obtain the following inequality:

$$
\forall \tau \in[0, T], \quad\|\mathbf{N}(\tau)\|_{n}^{2} \leq C_{1}\left(\mathbf{N}^{0}, \theta\right)+C\left(\varepsilon_{0}\right) \int_{0}^{\tau}\|\mathbf{N}(s)\|_{n}^{2} \mathrm{~d} s .
$$

According to the Gronwall's lemma and the remark on the norms equivalence, the element $\mathbf{N}$ of $K$ remains in a fixed bounded part of $\left(L^{\infty}\left(0, T ; \mathrm{H}^{1}(\Omega)\right)\right)^{n}$ and a fortiori, there exists a constant $\tilde{C}_{1}$ such that, for all $p \in] 1,+\infty]$

$$
\|\mathbf{N}\|_{\left(L^{p}\left(0, T ; \mathrm{H}^{1}(\Omega)\right)\right)^{n}} \leq \tilde{C}_{1}
$$

Then we use again inequality (1.5) in order to obtain (1.3). 


\subsection{A fixed point method}

The previous estimates prove that the functions set $\mathbf{N}=\mathbf{N}\left(f_{1}, f_{2}, \ldots, f_{n}\right)$ remains in a fixed bounded part of $X=\left(L^{\infty}\left(0, T ; \mathrm{H}^{1}(\Omega)\right) \cap H^{1}(Q)\right)^{n}$, independently of $\mathbf{f}$, when $\mathbf{f}$ covers $\left(H^{1}(Q)\right)^{n}$.

\section{Notations and strategy}

In order to use a fixed point method, we search a Banach space with separable dual space (in order to use Th. III.25', pp. 50 of [7]). Moreover, in order to have compacity result for the weak topology, we introduce the reflexive space for $p_{0}$ fixed in $\left[2,+\infty\left[X_{p_{0}}=\left\{L^{p_{0}}\left(0, T ; \mathrm{H}^{1}(\Omega)\right) \cap H^{1}(Q)\right\}^{n}\right.\right.$ and

$\mathcal{K}=\left\{\mathbf{V} \in X_{p_{0}},\|\mathbf{V}\|_{X_{p_{0}}} \leq C\right.$, where $C=\sqrt{\tilde{C}_{1}^{2}+C_{2}^{2}}, \mathbf{V}(0,)=.\mathbf{N}^{0}, \mathbf{V}(t,.) \in K$ a.e.in $\left.t\right\}$, the bounded part relative to the previous lemma.

From the consideration of the application $\Im_{p_{0}}: \mid \begin{aligned} & X_{p_{0}} \rightarrow X_{p_{0}} \\ & \mathbf{f} \rightarrow \mathbf{N}\end{aligned}$ and from the previous calculus, one can easily prove that the set $\mathcal{K}$ is stable by the application $\Im_{p_{0}}$. Moreover, $\mathcal{K}$ is a convex closed bounded nonempty set of $X_{p_{0}}$ and thus weakly compact of $X_{p_{0}}$.

Proposition 1.2. The application $\Im_{p_{0}}$ admits a fixed point which is solution of the problem (0.3).

Proof. We consider the application $\Im_{p_{0}}$ when $X_{p_{0}}$ is endowed with a weak topology $\sigma\left(X_{p_{0}},\left(X_{p_{0}}\right)^{\prime}\right)$, that gives a structure of topological locally convex separated vectorial space. In order to use a "fixed point method", one hast just to prove that $\Im_{p_{0}}$ is a weakly-weakly sequentially continuous from $\mathcal{K}$ to $\mathcal{K}$. So we consider a sequence $\left(\mathbf{f}^{q}\right)_{q \in N}$ of elements from $X_{p_{0}}$ that weakly converges in $X_{p_{0}}$ to $\mathbf{f}$.

The functions $\mathbf{N}^{q}$ and $\partial_{t} \mathbf{N}^{q}$ remaining respectively in some bounded fixed parts of $\left(L^{p_{0}}\left(0, T ; \mathrm{H}^{1}(\Omega)\right)\right)^{n}$ and of $\left(L^{2}(Q)\right)^{n}$, we can extract some weakly convergent subsequences of suffix $q_{k}$. Thus

$$
\exists \mathbf{N} \in X_{p_{0}}, \text { tel que } \mathbf{N}^{q_{k}} \stackrel{X_{p_{0}}}{\rightarrow} \mathbf{N} \text { et } \mathbf{N} \in \mathcal{K} .
$$

It remains to prove that

$$
\mathbf{N}=\Im_{p_{0}}(\mathbf{f})
$$

Now, for all $q_{k} \in \mathbb{N}$, the linear associated problems $\left(P_{\text {lin }}\left(f_{q_{k}}\right)\right)$ are written under their variationnal formulations by $\mathbf{N}^{\mathbf{q}_{\mathbf{k}}} \in \mathcal{K}$ and $\forall \mathbf{V} \in \mathcal{V}^{n}$, a.e. in $t$,

$$
\left\{\begin{array}{l}
\int_{\Omega} K_{i j}\left(f_{q_{k}}\right) \partial_{t} N_{j}^{q_{k}} V_{i} \mathrm{~d} x+\int_{\Omega} D_{i j} \vec{\nabla} N_{j}^{q_{k}} \cdot \vec{\nabla} V_{i} \mathrm{~d} x+\int_{\Omega} \mu^{\prime}\left(\left(f_{q_{k}}\right)_{i}\right) V_{i} \vec{\nabla} N_{i}^{q_{k}} \cdot \mathbf{U} \mathrm{d} x \\
+\int_{\Omega} \sigma^{i}\left(f_{q_{k}}\right) V_{i} \Delta \theta \mathrm{d} x+\int_{\Omega}\left(\mu_{i}^{l}\left(f_{q_{k}}\right) \frac{\partial N_{l}^{q_{k}}}{\partial x_{j}}\right) V_{i} \frac{\partial \theta}{\partial x_{j}} \mathrm{~d} x=0 .
\end{array}\right.
$$

With the help of the compacity of the embedding from $H^{1}(Q)$ to $L^{2}(Q)$ (Rellich-Kondrachoff's theorem)

$$
\mathbf{f}_{q_{k}} \stackrel{\mathcal{L}^{4}-\text { a.e. in } Q}{\longrightarrow} \mathbf{f}
$$

and, as $\tilde{K}$ is a matrix of regular functions, we have:

$$
K_{i j}\left(\mathbf{f}_{q_{k}}\right) V_{i} \stackrel{\mathcal{L}^{4}-\text { a.e.in } Q}{\longrightarrow} K_{i j}(\mathbf{f}) V_{i} .
$$


Using Lebesgue's dominated convergence theorem, and considering in $L^{2}(Q)$ the product of strong and weak convergences, we obtain:

$$
\int_{\Omega} K_{i j}\left(\mathbf{f}_{q_{k}}\right) \partial_{t} N_{j}^{n_{k}} V_{i} \mathrm{~d} x \stackrel{k \rightarrow \infty}{\longrightarrow} \int_{\Omega} K_{i j}(\mathbf{f}) \partial_{t} N_{j} V_{i} \mathrm{~d} x .
$$

We use the same argument for the other integrals with the essential fact here that the state functions $\left(\mu^{\prime}, \frac{\partial \sigma^{i}}{\partial x_{k}}\right.$, etc. $)$ are bounded and continuous. The trace application in $t=0$ being strongly continuous from $X_{p_{0}}$ to $L^{2}(\Omega)$ and linear, it is weakly-weakly continuous and thus

$$
\mathbf{N}(0, .)=\mathbf{N}^{0} .
$$

We obtain, passing to the limit, for all $\mathbf{V}$ in $\mathcal{V}^{n}$ and almost everywhere on $] 0, T[: \mathbf{N} \in \mathcal{K}$ and

$$
\left\{\begin{array}{c}
\int_{\Omega} K_{i j}(\mathbf{f}) \partial_{t} N_{j} V_{i} \mathrm{~d} x+\int_{\Omega} D_{i j} \vec{\nabla} N_{j} \cdot \vec{\nabla} V_{i} \mathrm{~d} x+\int_{\Omega} \mu^{\prime}\left(f_{i}\right) V_{i} \vec{\nabla} N_{i} \cdot \mathbf{U} \mathrm{d} x \\
+\int_{\Omega} \sigma^{i}(\mathbf{f}) V_{i} \Delta \theta \mathrm{d} x+\int_{\Omega}\left(\mu_{i}^{l}(\mathbf{f}) \frac{\partial N_{l}}{\partial x_{j}}\right) V_{i} \frac{\partial \theta}{\partial x_{j}} \mathrm{~d} x=0 \\
\mathbf{N}(0, .)=\mathbf{N}^{0}(.)
\end{array}\right.
$$

It follows that, according to the uniqueness property,

$$
\mathbf{N}=\Im_{p_{0}}(\mathbf{f})
$$

The cluster point $\Im_{p_{0}}(\mathbf{f})$ being independent of the extracted subsequence (because of the uniqueness of the solution of the linearized parabolic problem $\left(P_{\text {lin }}(\mathbf{f})\right)$, we deduce that the entire sequence $\left(\mathbf{N}^{q}\right)_{q \in N}$ converges weakly in $X_{p_{0}}$ to $\mathbf{N}=\Im_{p_{0}}(\mathbf{f})$. Then, the application $\Im_{p_{0}}$ is weakly-weakly sequentially continuous from $\mathcal{K}$ in $\mathcal{K}$, which is weakly compact. According to the Schauder - Tychonov lemma, $\Im_{p_{0}}$ admits a fixed point noted by $\mathbf{N}$. Hence, because for all $i \in\{1, \ldots, n\}$

$$
\left\{\begin{array}{l}
\sigma^{i}(\mathbf{N}) \Delta \theta+\left(\mu_{i}^{k}(\mathbf{N}) \frac{\partial N_{k}}{\partial x_{j}}\right) \frac{\partial \theta}{\partial x_{j}}=\operatorname{div}\left(\sigma^{i}(\mathbf{N}) \vec{\nabla} \theta\right), \\
\mu^{\prime}\left(N_{i}\right) \mathbf{U} \cdot \vec{\nabla} N_{i}=\operatorname{div}\left(\mu\left(N_{i}\right) \mathbf{U}\right), \quad \operatorname{car} \operatorname{div}(\mathbf{U})=0
\end{array}\right.
$$

which is a justification a posteriori of the formulation of the paralinearized problem, we can state: $\exists \mathbf{N} \in\left(L^{p_{0}}\left(0, T ; \mathrm{H}^{1}(\Omega)\right) \cap H^{1}(Q)\right)^{n}, \mathbf{N}(t) \in \mathcal{K}$ a.e. in $t$, such that, a.a. $t \in[0, T], \forall \mathbf{V} \in\left(L^{2}(0, T ; \mathcal{V})\right)^{n}$,

$$
(\mathbf{P})\left\{\begin{array}{c}
\left(\tilde{\mathbf{K}}(\mathbf{N}) \partial_{t} \mathbf{N}, \mathbf{V}\right)+d(\mathbf{N}, \mathbf{V})+U(\mathbf{N}, \mathbf{V})+\sigma(\mathbf{N}, \theta, \mathbf{V})=0 \\
\mathbf{N}(0, .)=\mathbf{N}^{0} .
\end{array}\right.
$$

Then, for $p_{0}$ taken in $[2,+\infty[$,

$$
\Im_{p_{0}}\left(X_{p_{0}}\right) \subset X \subset X_{p_{0}}
$$

and thus, a fortiori, all the fixed points of the application $\Im_{p_{0}}$, and in this case, $\mathbf{N}$ remains in $\left(L^{\infty}\left(0, T ; \mathrm{H}^{1}(\Omega)\right)\right)^{n}$. 
The result of the Proposition 1.2 holds in the following regularity frame:

$$
\mu \in W^{1,+\infty}(\mathbb{R}), \sigma^{i} \in W^{1,+\infty}\left(\mathbb{R}^{n}\right) .
$$

Indeed, we know that, using mollifiers, each function in $W^{1,+\infty}(\mathbb{R})$ can be approached by sequence of elements in $C^{\infty}(\mathbb{R}) \cap W^{1,+\infty}(\mathbb{R})$. The result of the proposition 1.2 remaining true for this type of functions, one can approach $\mu$ (resp. $\left(\sigma^{i}\right)$ ) by a sequence of elements in $C^{1}\left(\mathbb{R}^{n}\right) \cap W^{1,+\infty}\left(\mathbb{R}^{n}\right)$ ) that have the same Lipschitz module. We conclude, passing to the limit, and observing that, according to the proposition 1.1, the a priori estimates remain true, as they can be bounded independently of the considered state functions by this way of regularization.

\section{Physical admissibility of the SOlution}

Assumption 3. We suppose in this section that the following assumption is satisfied:

$$
(H)\left\{\begin{array}{l}
\forall(i, j), \forall\left(x_{1}, \ldots, x_{i-1}, 0, x_{i+1}, \ldots, x_{n}\right) \in\left(\mathbb{R}_{+}\right)^{n}, \frac{\partial k^{i}}{\partial x_{j}}\left(x_{1}, \ldots, x_{i-1}, 0, x_{i+1}, \ldots, x_{n}\right)=0, \\
\forall(i, j), \quad D_{i j}=D_{i j} \delta_{i j} \text { (diagonal diffusive tensor). }
\end{array}\right.
$$

Proposition 2.1. Under the previous assumption $(H)$, the solution of the problem $(P)$ verifies the property of physical admissibility:

$$
\forall \tau \in[0, T], \forall i \in\{1, \ldots, n\}, N_{i}(\tau, .) \geq 0, £^{3}-\text { a.e.in } \Omega .
$$

The proof is based on the fact that the space is stable by null truncature at the origin and allows the choice of the test function $\mathbf{V}=-\mathbf{N}^{-}$.

Remark 2.1. For some less elaborated models where the sum $\sum_{j=1}^{n} N_{j}$ is taken a priori as constant, equal to $\mathcal{N}_{0}$ (in order to fix ideas), the same method leads to the property

$$
\forall \tau \geq 0, \forall i \in\{1, \ldots, n\}, \quad N_{i}(\tau, .) \leq \mathcal{N}_{0} \text { a.e. in } \Omega .
$$

Generally, each method developed herein holds when the Soret coefficients $S_{t}^{i}$ are "Soret laws" (as functions of some weight fractions), as soon as there is a separated Lipschitz dependence on each variable $N_{i}$.

In the mono-dimensional case (capillary column), the Ascoli Lemma allows to set a more precise result; this is done in

Proposition 2.2 (Unidirectional moves, capillary tube case). When $\Omega$ is a bounded part of $\mathbb{R}$, i.e. $\Omega=] 0, L[$ and $\Gamma_{e}=\{0\}, \Gamma_{s}=\{L\}, \Gamma_{l}=\emptyset$, we prove that

$\forall i \in\{1, \ldots, n\}, N_{i} \in C^{0}([0, T] \times[0, L])=C^{0}(\bar{Q})$. Consequently,

$$
\exists M \geq 0, \forall i \in\{1, \ldots, n\}, \forall(t, x) \in \bar{Q}, \quad 0 \leq N_{i}(t, x) \leq M .
$$

Thus, the solutions are physically admissible by this property, deduced from the model.

\section{Uniqueness AND STABILITy of THE SOlUtion}

In this part, we prove the uniqueness of the solution using a duality technique, i.e. by looking for test-functions that allow to conclude. This technique, recently used by Diaz for Boussinesq-like problems (cf. [10] and [11]), reduces the uniqueness question to the study of the existence of a solution to the dual problem. We detail this transposition method here. 
Proposition 3.1. In order to prove uniqueness of the solution of the problem $(P)$, one has to prove the existence of a solution of a dual linear problem $\left(P^{\prime}\right)$.

Proof. Considering one by one each term of the equation, we denote by $\mathbf{N}=\left(\begin{array}{l}N_{1} \\ \ldots \\ N_{n}\end{array}\right)$ et $\hat{\mathbf{N}}=\left(\begin{array}{c}\hat{N}_{1} \\ \ldots \\ \hat{N}_{n}\end{array}\right)$ two possible solutions of $(P)$. We subtract the two equations verified by each solution to obtain:

$$
\left\{\begin{array}{c}
\left(\tilde{\mathbf{K}}_{i}(\mathbf{N}) \partial_{t} N_{j}-\tilde{\mathbf{K}}_{i}(\hat{\mathbf{N}}) \partial_{t} \hat{N}_{j}, \zeta\right)+\left(d_{i}(\mathbf{N}, \zeta)-d_{i}(\hat{\mathbf{N}}, \zeta)\right) \\
+\left(U_{i}(\mathbf{N}, \zeta)-U_{i}(\hat{\mathbf{N}}, \zeta)\right)+\left(\sigma_{i}(\mathbf{N}, \theta, \zeta)-\sigma_{i}(\hat{\mathbf{N}}, \theta, \zeta)\right)=0
\end{array}\right.
$$

where $\zeta$ is a test function of $\left(L^{2}(0, T ; \mathcal{V})\right)^{n}$ to be precised, and applications $d, U, \sigma$ are taken without being summed on $i$. Let's examine one to one each term of this equation, after having integrated them on $[0, T]$ :

$$
\begin{aligned}
I n t_{1}^{i}= & \int_{0}^{T}\left(\tilde{\mathbf{K}}_{i}(\mathbf{N}) \partial_{t} N_{j}-\tilde{\mathbf{K}}_{i}(\hat{\mathbf{N}}) \partial_{t} \hat{N}_{j}, \zeta\right) \mathrm{d} t \\
& \stackrel{\text { Green }}{=}-\int_{Q}\left[\kappa^{i}\left(N_{1}, . ., N_{n}\right)-\kappa^{i}\left(\hat{N}_{1}, . ., \hat{N}_{n}\right)\right] \partial_{t} \zeta_{i} \mathrm{~d} x \mathrm{~d} t
\end{aligned}
$$

for each function $\zeta$ verifying $\zeta_{i}(T)=0$ and because $\left[\kappa^{i}\left(N_{1}, . ., N_{n}\right)-\kappa^{i}\left(\hat{N}_{1}, . ., \hat{N}_{n}\right)\right](0)=\kappa^{i}\left(N^{0}\right)-\kappa^{i}\left(N^{0}\right)=0$. Thus,

$$
\operatorname{Int} t_{1}^{i}=-\int_{Q}\left[\sum_{j \in\{1 . . n\}}\left(\kappa^{i}\left(\hat{N}_{1}, . ., \hat{N}_{j-1}, N_{j}, . ., N_{n}\right)-\kappa^{i}\left(\hat{N}_{1}, . ., \hat{N}_{j}, N_{j+1}, . ., N_{n}\right)\right)\right] \partial_{t} \zeta_{i} \mathrm{~d} x \mathrm{~d} t
$$

Writing

$$
\kappa^{i}\left(\hat{N}_{1}, . ., \hat{N}_{j-1}, N_{j}, . ., N_{n}\right)-\kappa^{i}\left(\hat{N}_{1}, . ., \hat{N}_{j}, N_{j+1}, . ., N_{n}\right)=\left(N_{j}-\hat{N}_{j}\right) \kappa_{j}^{i}(t, x)
$$

we define on the $£^{4}$-measurable sets $\mathcal{E}_{j}=\left\{(t, x) \in Q\right.$ such that $\left.N_{j} \neq \hat{N}_{j}\right\}$ and $Q \backslash \mathcal{E}_{j}$

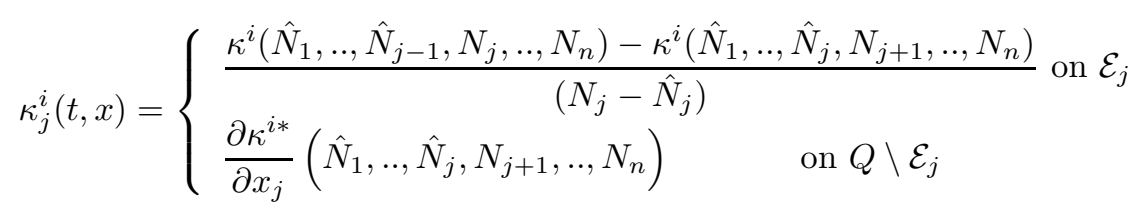

and $\frac{\partial \kappa^{i *}}{\partial x_{j}}$ is a bounded borelian representative (in it's Lebesgue class) of the derivative (in the classic sense), defined almost everywhere, of the Lipschtiz function $x_{j} \rightarrow \kappa^{i}\left(\ldots, x_{j}, \ldots\right)$ (Rademacher's theorem). Thus, we obtain the following expression for $I n t_{1}^{i}$ :

$$
\text { Int } t_{1}^{i}=-\int_{Q}\left[\sum_{j \in\{1 . . n\}}\left(N_{j}-\hat{N}_{j}\right) \kappa_{j}^{i}(t, x)\right] \partial_{t} \zeta_{i} \mathrm{~d} x \mathrm{~d} t
$$


Then, by summing the equations relative to each component, i.e. by summing on $i \in\{1 . . n\}$, we obtain:

$$
\operatorname{Int}_{1}=-\int_{Q} \sum_{j \in\{1 . . n\}}\left(\left(N_{j}-\hat{N}_{j}\right)\left[\sum_{i \in\{1 . . n\}} \kappa_{j}^{i}(t, x) \partial_{t} \zeta_{i}\right]\right) \mathrm{d} x \mathrm{~d} t
$$

We use the same technique for the third and the fourth terms of the equation (3.1), having first defined the functions

$$
\mu^{*}(t, x)= \begin{cases}\frac{\mu\left(N_{i}\right)-\mu\left(\hat{N}_{i}\right)}{N_{i}-\hat{N}_{i}} & \text { on } \mathcal{E}_{j} \\ \mu^{\prime *}\left(N_{i}\right) & \text { on } Q \backslash \mathcal{E}_{j}\end{cases}
$$

and

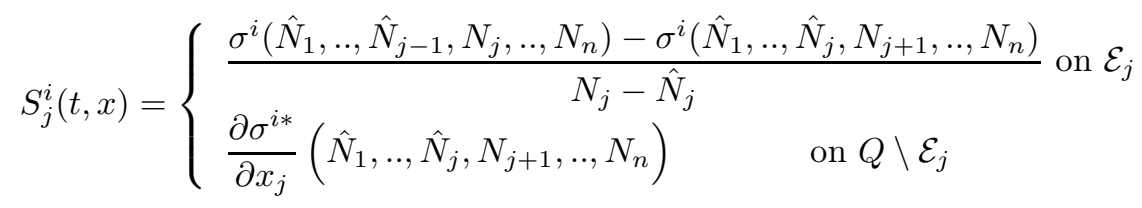

$\mu^{\prime *}$ and $\frac{\partial \sigma^{i *}}{\partial x_{j}}$ being, according to the same principle, some bounded borelian representatives of the derivatives of $\mu$ and of $\sigma^{i}$. Let's have a look to the second term of the equation (3.1):

$$
\begin{aligned}
\text { Int }_{2}^{i} & =\sum_{j \in\{1 . . n\}} D_{i j} \int_{\Omega} \vec{\nabla}\left(N_{j}-\hat{N}_{j}\right) \cdot \vec{\nabla} \zeta_{i} \mathrm{~d} x \\
& =-\sum_{j \in\{1 . . n\}} D_{i j} \int_{\Omega}\left(N_{j}-\hat{N}_{j}\right) \Delta \zeta_{i} \mathrm{~d} x+\sum_{j \in\{1 . . n\}} D_{i j} \int_{\partial \Omega}\left(N_{j}-\hat{N}_{j}\right) \frac{\partial \zeta_{i}}{\partial n} \mathrm{~d} \sigma \\
& =-\sum_{j \in\{1 . . n\}} D_{i j} \int_{\Omega}\left(N_{j}-\hat{N}_{j}\right) \Delta \zeta_{i} \mathrm{~d} x
\end{aligned}
$$

as soon as $\left.\frac{\partial \zeta_{i}}{\partial n}\right|_{\Gamma_{l}}=0$, because $N_{j}-\left.\hat{N}_{j}\right|_{\Gamma_{e} \cup \Gamma_{s}}=0$ by definition of the problem $(P)$. Then, we obtain, after having summed on $i \in\{1 . . n\}$ and integrated on $[0, T]$,

$$
I_{2} t_{2}=-\int_{Q} \sum_{j \in\{1 . . n\}}\left(N_{j}-\hat{N}_{j}\right)\left[\sum_{i \in\{1 . . n\}} D_{i j} \Delta \zeta_{i}\right] \mathrm{d} x \mathrm{~d} t .
$$

We obtain the following equation:

$$
(\dagger)\left\{\begin{array}{l}
\int_{Q}\left(\sum _ { j \in \{ 1 . . n \} } ( N _ { j } - \hat { N } _ { j } ) \left[-\sum_{i \in\{1 . . n\}} \kappa_{j}^{i}(t, x) \partial_{t} \zeta_{i}-\sum_{i \in\{1 . . n\}} D_{i j} \Delta \zeta_{i}\right.\right. \\
\left.\left.+\mu^{*}(t, x) \mathbf{U} \cdot \vec{\nabla} \zeta_{i}+\sum_{i \in\{1 . . n\}} S_{j}^{i}(t, x) \vec{\nabla} \theta \cdot \vec{\nabla} \zeta_{i}\right]\right) \mathrm{d} x \mathrm{~d} t=0
\end{array}\right.
$$


Let us consider in the following backward problem of unknown $\zeta=\left(\zeta_{i}\right)_{i}$ :

$$
\left(P^{\prime}\right)\left\{\begin{array}{l}
-\sum_{i \in\{1 . . n\}} \kappa_{j}^{i}(t, x) \partial_{t} \zeta_{i}-\sum_{i \in\{1 . . n\}} D_{i j} \Delta \zeta_{i}+\mu^{*}(t, x) \mathbf{U} \cdot \vec{\nabla} \zeta_{i}+\sum_{i \in\{1 . . n\}} S_{j}^{i}(t, x) \vec{\nabla} \theta \cdot \vec{\nabla} \zeta_{i}=N_{j}-\hat{N}_{j} \text { in } Q \\
\zeta_{j}(T)=0 \text { in } \Omega \\
\zeta_{j}=0 \text { on } \Gamma_{e} \cup \Gamma_{s}, \quad \frac{\partial \zeta_{j}}{\partial n}=0 \text { on } \Gamma_{l},(1 \leq j \leq n) .
\end{array}\right.
$$

The functions $\kappa_{j}^{i}, \mu^{*}, \quad S_{j}^{i}$ are in $L^{\infty}(Q)$ as soon as the state functions are Lipschitz ones against each variable, assumption that we will consider to be true. One can easily prove with the help of a priori estimates and the Lions theorem Lions ([7], p. 218) applied to vectorial equations that the problem $\left(P^{\prime}\right)$ admits a unique solution. The dependence of the solution during time on the initial state or on some parameters linked to the convective transfer is studied in the following proposition:

Proposition 3.2. The problem $(P)$ has a unique solution. Furthermore, the (nonlinear) application which associates $\mathbf{N}$ in $\left[L^{\infty}\left(0, T ; \mathrm{H}^{1}(\Omega)\right) \cap H^{1}(Q)\right]^{n}$ to $\mathbf{N}^{0}$ in $\mathrm{H}^{1}(\Omega)^{n}$ is a local Lipschitz one from $L^{2}(\Omega)^{n}$ to $L^{2}(Q)^{n}$. In the same way, the (nonlinear) application that associates $\mathbf{N}$ to $\left\{S_{t}^{i}\right\}_{1 \leq i \leq n}[$ resp. $\vec{\nabla} \theta]$ is a local Lipschitz one from $\mathbb{R}^{n}$ to $L^{2}(Q)^{n}\left[\right.$ resp. from $\mathrm{L}^{\infty}(\Omega)^{n}$ to $\left.\mathrm{L}^{2}(Q)^{n}\right]$.

Proof. Taking precisely for test function $\zeta$ the solution of the associated backward system $\left(P^{\prime}\right)$ and introducing the equation verified by $\zeta$ in the expression $(\dagger)$, we obtain immediately, according to the solutions regularity,

$$
\forall t \in[0, T], \mathbf{N}(t, .)=\hat{\mathbf{N}}(t, .) £^{3}-\text { a.e. in } \Omega .
$$

In order to prove the stability of the solution of the problem $(P)$, let us consider $\mathbf{N}$ (resp. $\hat{\mathbf{N}}$ ) the solution relative to $\mathbf{N}^{0}$ (resp. $\hat{\mathbf{N}}^{0}$ ). Then, using again the previous method and for the same choice of $\zeta$, we get

$$
\|\mathbf{N}-\hat{\mathbf{N}}\|_{\left(L^{2}(Q)\right)^{n}}^{2}=\sum_{i=1}^{n}\left(N_{i}^{0}-k_{i}\left(\mathbf{N}^{0}\right)-\hat{N}_{i}^{0}+k_{i}\left(\hat{\mathbf{N}}^{0}\right), \zeta^{i}(0)\right)_{L^{2}(\Omega)} .
$$

As, a.e. in $t$ and a.e. in $\Omega$, and for $i \in\{1, \ldots, n\}$

$$
\left|N_{i}^{0}-\hat{N}_{i}^{0}-\left(k_{i}\left(\mathbf{N}^{0}\right)-k_{i}\left(\hat{\mathbf{N}}^{0}\right)\right)\right| \leq\left|N_{i}^{0}-\hat{N}_{i}^{0}\right|+\left(\sum_{j=1}^{n}\left|N_{j}^{0}-\hat{N}_{j}^{0}\right|\left\|\frac{\partial k_{i}}{\partial x_{j}}\right\|_{L^{\infty}\left(\mathbb{R}^{n}\right)}\right)
$$

and, with the help of general results on the linear parabolic equations on the continuous dependence of the solutions on the data ( $c f$. Dautray and Lions [8], Vol. 8, Chap. XVIII, $\S .3$ and 4), (here $\zeta$ is a solution relatively to an initial vanishing data and a sink term equal to $\mathbf{N}-\hat{\mathbf{N}}$ ), there exists a constant $C^{*}$, depending a priori on $\Omega, T, \mathbf{U}, \theta$, and on the state functions, such that:

$$
|\zeta(\mathbf{0})|_{L^{2}(\Omega)^{n}} \leq|\zeta|_{C^{0}\left([0, T] ; L^{2}(\Omega)^{n}\right)} \leq C^{*}\|\mathbf{N}-\hat{\mathbf{N}}\|_{\left(L^{2}(Q)\right)^{n}}^{2}
$$

Thus, according to $(*),(* *)$, and $(* * *)$ and due to the Cauchy-Schwarz inequality, there exists a constant $C$ (which depends mainly on the Lipschitz constant $C^{*}$ and on the Lipschitz modules of the partial functions $\left.x_{j} \longrightarrow k_{i}\left(\ldots, x_{j}, \ldots\right)\right)$, such as

$$
\|\mathbf{N}-\hat{\mathbf{N}}\|_{\left(L^{2}(Q)\right)^{n}} \leq C\left|\mathbf{N}^{0}-\hat{\mathbf{N}}^{0}\right|_{L^{2}(\Omega)^{n}} .
$$

The other properties are obtained with the same transposition method. 


\section{A NUMERICAL SCHEME}

In the following part, $n_{c}$ will denote the number of unknowns (i.e. the number of components in the fluid) in order to avoid any confusion between this one and the time discretization suffix.

We present here a numerical scheme based on a mixed formulation $[22,23]$ of the problem for which we give numerical analysis.

First, we describe briefly the adopted mesh and present the different spaces and notations used in the analysis. Then we prove the results on the approximation error made in the stationary problem. Finally we study the evolutive problem for which we demonstrate existence and uniqueness of a solution and present convergence and consistence results for the numerical scheme.

The main difficulty in this numerical study remains in the verification of three Inf-Sup conditions associated with the used mixed formulation. The choice of the discretization spaces likely to verify these conditions must be done with caution. Moreover, we treat a system and no more a scalar equation. We will see that under ad $h o c$ assumptions on diffusive and adsorption tensors (which are close to the reality), it is possible to prove the method's convergence. Finally, we link the proposed discretization with the finite volumes method.

Remark 4.1. Here is detailed the numerical analysis of a scheme in the case of low Peclet numbers (situation where the diffusive phenomena dominate the convection, which is true for viscous fluids submitted to natural convection in deposits).

In a first time, we are interested in the following problem:

$$
-\operatorname{div}\left(\tilde{\tilde{\mathbf{D}}}(\nabla \mathbf{N})^{\top}\right)+\tilde{\tilde{\mathbf{K}}} \mathbf{N}=f(\mathbf{N}) \Delta \theta
$$

which is rewritten, $\forall i \in\left\{1 . . n_{c}\right\}$,

$$
-\sum_{1 \leq l \leq n_{c}} D_{i l} \Delta N_{l}+\sum_{1 \leq j \leq n_{c}} K_{i j} N_{j}=f_{i}(\mathbf{N}, x) .
$$

We define then $\tilde{\tilde{p}}=\tilde{\tilde{D}}(g \tilde{r a} d \mathbf{N})^{\top}$. An equivalent problem is given by, $\forall i \in\left\{1 . . n_{c}\right\}$,

$$
-\operatorname{div}\left(p_{i}\right)+\sum_{1 \leq j \leq n_{c}} K_{i j} N_{j}=f_{i}(\mathbf{N}, x)
$$

\subsection{Notations}

We modelize the medium by a planar rectangular column, and construct a regular rectangular mesh. Let us introduce the following spaces:

$$
W_{1}=(H(\operatorname{div}, \Omega))^{n_{c}}, W_{2}=\left(\left(L^{2}(\Omega)\right)^{2}\right)^{n_{c}}, M_{1}=\left(\mathrm{H}_{0}^{1}(\Omega)\right)^{n_{c}}, M_{2}=L^{2}(\Omega)
$$

endowed with their usual norms, to which we associate the discrete spaces

$$
W_{1 h} \subset W_{1}, W_{2 h} \subset W_{2}, M_{1 h} \subset M_{1}, M_{2 h} \subset M_{2} .
$$

Then, we define the spaces

- $P_{0}(K)$ the space of constant functions on $K$

- $P_{1,0}(K)$ the space of linear functions (against the first space variable) on $K$

- $P_{0,1}(K)$ the space of linear functions (against the second space variable) on $K$

- $Q_{1}(K)$ the space of linear functions (against each space variable) on $K$. 

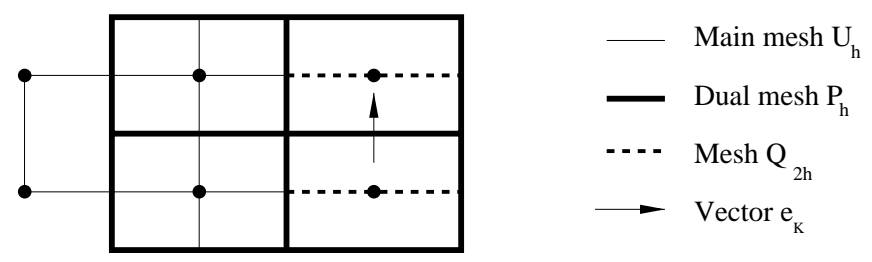

FiguRE 1. Visualization of the different meshes.

We will denote by $\left(P_{h}\right)_{h}$ (resp. $\left.\left(U_{h}\right)_{h}\right)$ the family of triangulations associated with $W_{1 h}$ (resp. $\left.M_{1 h}\right)$. Then, we construct the meshes $Q_{1 h}$ (resp. $Q_{2 h}$ ) by joining the middles of the horizontal (resp. vertical) faces of $P_{h}$. Then, we denote by $K$ a mesh of $P_{h}, K^{*}$ an element of $U_{h}$ and $K^{\sharp}$ an element of $Q_{h}=Q_{1 h} \cup Q_{2 h}$. These different meshes are illustrated in Figure 1.

Then, we can give the exact definition of the approximation spaces

$$
\begin{aligned}
& M_{1 h}=\left\{u^{h} \in\left(\mathrm{H}_{0}^{1}(\Omega)\right)^{n_{c}} ; \forall K^{*} \in U_{h}, u_{i}^{h} \in Q_{K^{*}}\left(K^{*}\right)\right\} \\
& M_{2 h}=\left\{u^{h} \in L^{2}(\Omega) ; \forall K \in P_{h},\left.u^{h}\right|_{K} \in P_{0}(K)\right\} . \\
& W_{1 h}=\left\{p^{h} \in W_{1}, \forall K \in P_{h}, p_{\left.i\right|_{K} ^{h}}^{h} \in P_{1,0}(K) \times P_{0,1}(K)\right\} \\
& W_{2 h}=\operatorname{Vect}\left\{e_{K^{\sharp}}, K^{\sharp} \in Q_{h}\right\}^{n_{c}} .
\end{aligned}
$$

It is possible to define the following applications:

$$
\begin{array}{rlrl}
\forall \tilde{q} & \in\left(\mathrm{L}^{2}(\Omega)^{2}\right)^{n_{c}}, p_{i} \in H(\operatorname{div}, \Omega)^{n_{c}}, v \in \mathrm{L}^{2}(\Omega), \mathbf{N} \in\left(H_{0}^{1}(\Omega)\right)^{n_{c}} \\
& \bullet m^{i}(\tilde{p}, \tilde{q})=\int_{\Omega} \tilde{p}_{i} \tilde{q}_{i} \mathrm{~d} x & \bullet d^{i}(v, \mathbf{N})=-\sum_{1 \leq j \leq n_{c}} K_{i j} \int_{\Omega} N_{j} v \mathrm{~d} x \\
\bullet & b^{i}(v, \tilde{p})=\int_{\Omega} v \operatorname{div}\left(\tilde{p}_{i}\right) \mathrm{d} x & \bullet a^{i}(\mathbf{N}, \tilde{q})=-\sum_{1 \leq j \leq n_{c}} D_{i j} \int_{\Omega} \tilde{\nabla} N_{j} \cdot \tilde{q_{i}} \mathrm{~d} x \\
\bullet & l^{i}(v)=-\int_{\Omega} f_{i} v \mathrm{~d} x
\end{array}
$$

with $\mathbf{f}=\left(f_{i}\right)_{i \in\left\{1 . . n_{c}\right\}}$ being a function of $L^{2}(\Omega)^{n_{c}}$.

We associate to this problem the following mixed primal dual formulation:

$\forall i \in\left\{1 . . n_{c}\right\}$, find $\left(\tilde{p}_{i}, N_{i}\right) \in H(\operatorname{div}, \Omega) \times \mathrm{H}_{0}^{1}(\Omega)$ solution of

$$
\left\{\begin{array}{l}
\forall \tilde{q} \in\left(L^{2}(\Omega)^{2}\right)^{n_{c}}, \quad m^{i}(\tilde{p}, \tilde{q})+a^{i}(\mathbf{N}, \tilde{q})=0 \\
\forall v \in L^{2}(\Omega), \quad b^{i}(v, \tilde{p})+d^{i}(v, \mathbf{N})=l^{i}(v)
\end{array}\right.
$$

We introduce the operators $R$ and $R^{*}$ to which we give the expression on $W_{1 h}$ and $M_{1 h}$

$$
\forall \tilde{p}^{h} \in W_{1 h}, R\left(\tilde{p}^{h}\right) \in W_{2 h} \text { and } R\left(\tilde{p}^{h}\right)=\left.\sum_{K^{\sharp} \in Q_{h}}\left(\tilde{p}^{h} \cdot e_{K^{\sharp}}\right)\right|_{\Gamma_{K^{\sharp}}} e_{K^{\sharp}}
$$

where $\Gamma_{K^{\sharp}}$ is the edge of an element $K$ of $P_{h}$ included in $K^{\sharp}$ of $Q_{h}$,

$$
\forall u^{h} \in M_{1 h}, R^{*}\left(u^{h}\right) \in W_{2 h} \text { and } R^{*}\left(u^{h}\right)=\left.\sum_{K^{\sharp} \in Q_{h}}\left(\tilde{\nabla} u^{h} \cdot e_{K^{\sharp}}\right)\right|_{\sigma_{K^{\sharp}}} e_{K^{\sharp}}
$$


where $\sigma_{K^{\sharp}}$ is the edge of an element $K^{*}$ of $U_{h}$ included in $K^{\sharp}$ of $Q_{h}$. Then, we introduce the numerical approximation spaces, defined for all $i \in\left\{1 . . n_{c}\right\}$

$$
m_{h}^{i}(\tilde{p}, \tilde{q})=\int_{\Omega} R\left(\tilde{p}_{i}\right) \tilde{q_{i}} \mathrm{~d} x \text { and } d_{h}^{i}(v, \mathbf{N})=\sum_{1 \leq j \leq n_{c}} K_{i j} \int_{\Omega} \Pi_{0, h}\left(N_{j}\right) v \mathrm{~d} x .
$$

and the spaces $W_{1 h}^{0}$ and $W_{2 h}^{0}$ defined by

$$
\begin{aligned}
& W_{1 h}^{0}=\left\{\tilde{p}^{h} \in W_{1 h}, \forall v^{h} \in M_{2 h}, \quad b^{i}\left(v^{h}, \tilde{p}^{h}\right)=0\right\} \\
& W_{2 h}^{0}=\left\{\tilde{q}^{h} \in W_{2 h}, \forall \tilde{p}^{h} \in W_{1 h}^{0}, m_{h}^{i}\left(\tilde{p}^{h}, \tilde{q}^{h}\right)=0\right\} .
\end{aligned}
$$

Consequently, we consider the following discrete problem

$$
\left(P_{h}\right)\left\{\begin{array}{l}
\text { Find }\left(\tilde{p}^{h}, \mathbf{N}^{h}\right) \in W_{1 h} \times M_{1 h} \text { such that } \\
\forall i \in\left\{1 . . n_{c}\right\},\left\{\begin{array}{l}
\forall \tilde{q}_{i}^{h} \in W_{2 h}, m_{h}^{i}\left(\tilde{p}^{h}, \tilde{q}^{h}\right)+a^{i}\left(\mathbf{N}^{h}, \tilde{q}^{h}\right)=0 \\
\forall v^{h} \in M_{2 h}, b^{i}\left(v^{h}, \tilde{p}^{h}\right)+d_{h}^{i}\left(v^{h}, \mathbf{N}^{h}\right)=l^{i}\left(v^{h}\right) .
\end{array}\right.
\end{array}\right.
$$

We give here two lemmas based on elementary calculus.

\section{Lemma 4.1.}

$$
\forall i \in\left\{1 . . n_{c}\right\}, \forall N_{i}^{h} \in M_{1 h},\left\|N_{i}^{h}-\Pi_{0, h}\left(N_{i}^{h}\right)\right\|_{0, \Omega} \leq C_{i} h\left\|N_{i}^{h}\right\|_{1, \Omega} .
$$

\section{Lemma 4.2.}

$$
\forall i \in\left\{1 . . n_{c}\right\}, \forall N_{i}^{h} \in M_{1 h},\left\|N_{i}^{h}\right\|_{0, \Omega} \leq C_{i}^{*}\left\|\Pi_{0, h}\left(N_{i}^{h}\right)\right\|_{0, \Omega} .
$$

\subsection{Results on the approximation error}

Let us consider the following non-homogeneous permanent problem:

$$
\left(P_{h}^{g}\right)\left\{\begin{array}{l}
\left(\tilde{p}^{h}, \mathbf{N}^{h}\right) \in W_{1 h} \times M_{1 h} \\
\forall i \in\left\{1 . . n_{c}\right\},\left\{\begin{array}{l}
\forall \tilde{q}^{h} \in W_{2 h}, m_{h}^{i}\left(\tilde{p}^{h}, \tilde{q}^{h}\right)+a^{i}\left(\mathbf{N}^{h}, \tilde{q}^{h}\right)=L^{i}\left(\tilde{q}^{h}\right) \\
\forall v^{h} \in M_{2 h}, b^{i}\left(v^{h}, \tilde{p}^{h}\right)=l^{i}\left(v^{h}\right) .
\end{array}\right.
\end{array}\right.
$$

where $L^{i}$ is defined by: $\tilde{q}^{h} \in W_{2 h} \longmapsto L^{i}\left(\tilde{q}^{h}\right)=\int_{\Omega} \tilde{g} \cdot \tilde{q}^{h} \mathrm{~d} x, \tilde{g}$ being an element of $L^{2}(\Omega)^{n_{c}}$.

As the Nicolaïdes theory shows [21], generalizing the Babuska-Brezzi theory, the study of such a system is based on the modification of three Inf-Sup conditions, given by

$$
\begin{gathered}
\inf _{\tilde{p}^{h} \in W_{1 h}^{0}} \sup _{\tilde{q}^{h} \in W_{2 h}} \frac{m_{h}^{i}\left(\tilde{p}^{h}, \tilde{q}^{h}\right)}{\left\|\tilde{p}^{h}\right\| \cdot\left\|\tilde{q}^{h}\right\|} \geq \alpha_{1}^{i}>0 \\
\inf _{\mathbf{N}^{h} \in M_{1 h}} \sup _{\tilde{q}^{h} \in W_{2 h}^{0}} \frac{a^{i}\left(\mathbf{N}^{h}, \tilde{q}^{h}\right)}{\left\|\mathbf{N}^{h}\right\| \cdot\left\|\tilde{q}^{h}\right\|} \geq \alpha_{2}^{i}>0 \\
\inf _{v^{h} \in M_{2 h}} \sup _{\tilde{p}^{h} \in W_{1 h}} \frac{b^{i}\left(v^{h}, \tilde{p}^{h}\right)}{\left\|\tilde{p}^{h}\right\| \cdot\left\|v^{h}\right\|} \geq \alpha_{3}^{i}>0 .
\end{gathered}
$$


The discretization spaces given previously are constructed in such a way that these three conditions are proved. For more details on the verification of these Inf-Sup conditions, one can refer to works of Thomas and Trujillo [26]. Then we prove the following result:

Lemma 4.3. The solution $\left(\tilde{p}^{h}, \mathbf{N}^{h}\right)$ of the problem $\left(P_{h}^{g}\right)$ verifies: $\exists C>0$, independent of $h$, such that

$$
\left\|\tilde{p}^{h}\right\|_{H(\mathrm{div}, \Omega)^{n_{c}}}+\left|\mathbf{N}^{h}\right|_{(1, \Omega)^{n_{c}}} \leq C\left\{\|f\|_{0, \Omega}+\|g\|_{0, \Omega}\right\} .
$$

Proof. The demonstration of this lemma is based on the consideration of particular test-functions $\tilde{q}^{h}=R^{*}\left(N^{h}\right)$ and $v^{h}=\Pi_{0, h}\left(N_{i}^{h}\right)$ in the formulation of the problem $\left(P_{h}^{g}\right)$. Then, we have

$$
m_{h}^{i}\left(\tilde{p}, R^{*}\left(\mathbf{N}^{h}\right)\right)+a^{i}\left(\mathbf{N}^{h}, R^{*}\left(\mathbf{N}^{h}\right)\right)+b^{i}\left(\Pi_{0, h} N_{i}^{h}, \tilde{p}\right)=L^{i}\left(R^{*}\left(\mathbf{N}^{h}\right)\right)+l^{i}\left(\Pi_{0, h} N_{i}^{h}\right) .
$$

The discrete Green's formula assures that

$$
m_{h}^{i}\left(\tilde{p}, R^{*}\left(\mathbf{N}^{h}\right)\right)=-b^{i}\left(\Pi_{0, h} N_{i}^{h}, \tilde{p}\right) .
$$

Thus, summing on $i \in\left\{1 . . n_{c}\right\}$, we obtain

$$
\sum_{i \in\left\{1 . . n_{c}\right\}} a^{i}\left(\mathbf{N}^{h}, R^{*}\left(\mathbf{N}^{h}\right)\right)=\sum_{i \in\left\{1 . . n_{c}\right\}}\left(L^{i}\left(R^{*}\left(\mathbf{N}^{h}\right)\right)+l^{i}\left(\Pi_{0, h} N_{i}^{h}\right)\right) .
$$

A Cauchy-Schwarz and Poincaré inequalities and the condition (4.1) allow to conclude that there exists a constant $C>0$ such that

$$
\forall i \in\left\{1 . . n_{c}\right\}, \quad\left|N_{i}^{h}\right|_{1, \Omega} \leq C\left\{\|f\|_{0, \Omega}+\|g\|_{0, \Omega}\right\} .
$$

We prove in a same way that taking for test-function $\tilde{q}^{h}=R\left(\tilde{p}^{h}\right)$ in the first equation of $\left(P_{h}^{g}\right)$, we obtain

$$
m_{h}^{i}\left(\tilde{p}^{h}, R\left(\tilde{p}^{h}\right)\right)+a^{i}\left(\mathbf{N}^{h}, R\left(\tilde{p}^{h}\right)\right)=0 ;
$$

with the previous result, we deduce that

$$
\forall i \in\left\{1 . . n_{c}\right\},\left\|p_{i}^{h}\right\|_{0, \Omega} \leq C\left\{\|f\|_{0, \Omega}+\|g\|_{0, \Omega}\right\} .
$$

And, finally, taking for test-function $v^{h}=\operatorname{div}\left(p_{i}^{h}\right)$ in the second equation of $\left(P_{h}^{g}\right)$, we have, using the lemma 4.1,

$$
\forall i \in\left\{1 . . n_{c}\right\},\left\|\operatorname{div}\left(p_{i}^{h}\right)\right\|_{0, \Omega} \leq C\left\{\|f\|_{0, \Omega}+\|g\|_{0, \Omega}\right\},
$$

that allows to conclude.

We are able to prove the result on approximation error given by the following theorem.

Theorem 4.1. Denoting $(\mathbf{N}, \tilde{p})$ the solution of problem $(P)$. If $(\mathbf{N}, \tilde{p}) \in\left(H^{2}(\Omega) \times\left(H^{1}(\Omega)\right)^{2}\right)^{n_{c}}$ and $\operatorname{div}(\tilde{p}) \in$ $\left(\mathrm{H}^{1}(\Omega)\right)^{n_{c}}$, then, $\exists C>0$ independent of $h$, such that,

$$
\left\|\tilde{p}-\tilde{p}^{h}\right\|_{H(\operatorname{div}, \Omega)^{n_{c}}}+\left|\mathbf{N}-\mathbf{N}^{h}\right|_{(1, \Omega)^{n_{c}}} \leq C h\left\{|\operatorname{div}(\tilde{p})|_{(1, \Omega)^{n_{c}}}+|\tilde{p}|_{(1, \Omega)^{n_{c}}}+\|\mathbf{N}\|_{(2, \Omega)^{n_{c}}}\right\} .
$$

Proof. We consider $\tilde{r}^{h}$ and $\mathbf{m}^{h}$ two elements of $W_{1 h}$ and $M_{1 h}$. We have, $\forall \tilde{q}^{h} \in W_{2 h}$,

$$
m_{h}^{i}\left(\tilde{p}^{h}-\tilde{r}^{h}, \tilde{q}^{h}\right)+a^{i}\left(\mathbf{N}^{h}-\mathbf{m}^{h}, \tilde{q}^{h}\right)=m^{i}\left(p-\tilde{r}^{h}, \tilde{q}^{h}\right)+a^{i}\left(\mathbf{N}-\mathbf{m}^{h}, \tilde{q}^{h}\right)+m_{h}^{i}\left(\tilde{r}^{h}, \tilde{q}^{h}\right)-m^{i}\left(\tilde{r}^{h}, \tilde{q}^{h}\right) .
$$


In a same way, $\forall v^{h} \in M_{2 h}$,

$$
b^{i}\left(v^{h}, \tilde{p}^{h}-\tilde{r}^{h}\right)+d_{h}^{i}\left(\mathbf{N}^{h}-\mathbf{m}^{h}, v^{h}\right)=b^{i}\left(v^{h}, \tilde{p}-\tilde{r}^{h}\right)-d_{h}^{i}\left(\mathbf{m}^{h}, v^{h}\right)+d^{i}\left(\mathbf{N}, v^{h}\right) .
$$

Thus, with lemma 4.3, we get, summing on $i$,

$$
\begin{aligned}
\left\|\tilde{p}^{h}-\tilde{r}^{h}\right\|_{H(\operatorname{div}, \Omega)^{n_{c}}}+\left|\mathbf{N}^{h}-\mathbf{m}^{h}\right|_{(1, \Omega)^{n_{c}}} \leq C\{ & \left\|\tilde{p}-\tilde{r}^{h}\right\|_{(0, \Omega)^{n_{c}}}+\left|\mathbf{N}-\mathbf{m}^{h}\right|_{(1, \Omega)^{n_{c}}} \\
& +\left\|R\left(\tilde{r}^{h}\right)-\tilde{r}^{h}\right\|_{(0, \Omega)^{n_{c}}}+\left\|\operatorname{div}\left(\tilde{p}-\tilde{r}^{h}\right)\right\|_{(0, \Omega)^{n_{c}}} \\
& \left.+\left\|\mathbf{N}-\Pi_{0, h}\left(\mathbf{m}^{h}\right)\right\|_{(0, \Omega)^{n_{c}}}\right\} .
\end{aligned}
$$

We easily deduce the result, using Lemma 4.1 and some triangular inequalities.

\subsection{Study of the evolutive problem}

In this part we study the initial evolutive problem. However, we place ourselves in the framework of the remark 4.1. This assumption, made to simplify the study, is justified in any situation where the mixture is highly diluted, i.e. each specie is low concentrated (so that it gives strong concentration gradients). In order to do a time discretization of the system, for a fixed time step $\Delta t=\frac{T}{N}$, we state $t_{n}=n \Delta t$ and we note $N_{i}(n \Delta t, \mathbf{x})=N_{i, n}(\mathbf{x}), \tilde{p}_{i}(n \Delta t, \mathbf{x})=\tilde{p}_{i, n}(\mathbf{x})$. Let us introduce the problem defined by the following variational formulation

$$
\left(P_{h}^{n}\right)\left\{\begin{array}{l}
\text { Find }\left(\tilde{p}_{n+1}^{h}, \mathbf{N}_{n+1}^{h}\right) \in W_{1 h} \times M_{1 h} \\
\forall i \in\left\{1 . . n_{c}\right\},\left\{\begin{array}{l}
\forall \tilde{q}^{h} \in W_{2 h}, m_{h}^{i}\left(\tilde{p}_{n+1}^{h}, \tilde{q}^{h}\right)+a^{i}\left(\mathbf{N}_{n+1}^{h}, \tilde{q}^{h}\right)=0 \\
\forall v^{h} \in M_{2 h}, b^{i}\left(v^{h}, \tilde{p}_{n+1}^{h}\right)+\frac{1}{\Delta t} d_{h}^{i}\left(v^{h}, \mathbf{N}_{n+1}^{h}-\mathbf{N}_{n}^{h}\right)=l^{i, n}\left(v^{h}\right) .
\end{array}\right.
\end{array}\right.
$$

Theorem 4.2. The problem $\left(P_{h}\right)$ admits a unique solution.

Proof. The proof of this theorem is essentially based on the fact that $\operatorname{dim}\left(W_{1 h}\right)+\operatorname{dim}\left(M_{1 h}\right)=\operatorname{dim}\left(W_{2 h}\right)+$ $\operatorname{dim}\left(M_{2 h}\right)$ which leads the study of the existence of a solution to the question of the uniqueness. Moreover, one has to consider the Nicolaïdes theory ( $c f .[21])$, and particularly one of the three "Inf-Sup" conditions given by the existence of a real constant $\alpha_{2}^{i}$ verifying

$$
\inf _{\mathbf{N}^{h} \in M_{1 h}} \sup _{\tilde{q}^{h} \in W_{2 h}^{0}} \frac{a^{i}\left(\mathbf{N}^{h}, \tilde{q}^{h}\right)}{\left\|\mathbf{N}^{h}\right\| \cdot\left\|\tilde{q}^{h}\right\|} \geq \alpha_{2}^{i}>0 .
$$

The proof of a such condition is based on the essential assumption of pseudo-ellipticity of the tensors $\tilde{\tilde{D}}$ and $\tilde{\tilde{K}}$, given by the the Hypothesis 1.(H). So, we consider the homogeneous problem of which we want to prove that its solution is zero. Taking the following test-functions,

$$
\tilde{q}_{i}^{h}=R^{*}\left(N_{i}^{h}\right) \text { and } v^{h}=\Pi_{0, h}\left(N_{i}^{h}\right),
$$

we obtain, summing the equations of $\left(P_{h}\right)$

$$
\forall 1 \leq i \leq n_{c},\left\{\begin{array}{l}
m_{h}^{i}\left(\tilde{p}, R^{*}\left(\mathbf{N}^{h}\right)\right)+a^{i}\left(\mathbf{N}^{h}, R^{*}\left(\mathbf{N}^{h}\right)\right) \\
+b^{i}\left(\Pi_{0, h}\left(N_{i}^{h}\right), \tilde{p}^{h}\right)+d_{h}^{i}\left(\Pi_{0, h}\left(N_{i}^{h}\right), \mathbf{N}^{h}\right)=0 .
\end{array}\right.
$$

Then we use a discrete Green's formula that we prove with a direct calculus element by element and which is given by

$$
m_{h}^{i}\left(\tilde{p}, R^{*}\left(\mathbf{N}^{h}\right)\right)=-b^{i}\left(\Pi_{0, h}\left(N_{i}^{h}\right), \tilde{p}^{h}\right),
$$


and thus, summing on each component

$$
\sum_{1 \leq i \leq n_{c}} a^{i}\left(\mathbf{N}^{h}, R^{*}\left(\mathbf{N}^{h}\right)\right)+\sum_{1 \leq i \leq n_{c}} d_{h}^{i}\left(\Pi_{0, h}\left(N_{i}^{h}\right), \mathbf{N}^{h}\right)=0 .
$$

As

$$
\sum_{1 \leq i \leq n_{c}} d_{h}^{i}\left(\Pi_{0, h}\left(N_{i}^{h}\right), \mathbf{N}^{h}\right)=\sum_{1 \leq i \leq n_{c}} K_{i j} \int_{\Omega} \Pi_{0, h}\left(N_{j}^{h}\right) \Pi_{0, h}\left(N_{i}^{h}\right) \mathrm{d} x
$$

we have, using Assumption 1.(H),

$$
\sum_{1 \leq i \leq n_{c}} d_{h}^{i}\left(\Pi_{0, h}\left(N_{i}^{h}\right), \mathbf{N}^{h}\right) \geq \alpha\left\|\Pi_{0, h}\left(\mathbf{N}^{h}\right)\right\|_{\left(W_{1, h}\right)}^{2} \geq 0
$$

and thus

$$
\sum_{1 \leq i \leq n_{c}} a^{i}\left(\mathbf{N}^{h}, R^{*}\left(\mathbf{N}^{h}\right)\right) \leq 0
$$

and, always with the help of direct consequences of 1.(H),

$$
\left|N_{i}^{h}\right|_{1, \Omega}=0, \forall i \in\left\{1 . . n_{c}\right\} \text {. }
$$

\subsection{Schema's consistence}

In this study, we first define the equivalent of a projection of the exact solution on the discrete space $W_{1 h} \times M_{1 h}$. Thus, we get at each time $t_{n}$, the couple $\left(\Phi \tilde{p}_{n}^{h}, \psi \mathbf{N}_{n}^{h}\right)$ using the following permanent problem

$$
\left\{\begin{array}{l}
\text { Find }\left(\Phi \tilde{p}_{n}^{h}, \psi \mathbf{N}_{n}^{h}\right) \in W_{1 h} \times M_{1 h} \\
\forall \tilde{q}^{h} \in W_{2 h}, \quad m_{h}^{i}\left(\Phi \tilde{p}_{n}^{h}, \tilde{q}^{h}\right)+a^{i}\left(\psi \mathbf{N}_{n}^{h}, \tilde{q}^{h}\right)=0 \\
\forall v^{h} \in M_{2 h}, \quad b^{i}\left(v^{h}, \Phi \tilde{p}_{n}^{h}\right)=b^{i}\left(v^{h}, \tilde{p}_{n}^{h}\right) .
\end{array}\right.
$$

This problem has already been studied in the previous paragraph; considering that

$$
\left\|\Phi \tilde{p}_{i, n}-\tilde{p}_{i, n}\right\|_{H(\operatorname{div}, \Omega)}+\left\|\psi N_{i, n}-N_{i, n}\right\| \leq C h,
$$

it remains to study the error between $\left(\Phi \tilde{p}_{n}^{h}, \psi \mathbf{N}_{n}^{h}\right)$ and $\left(\tilde{p}_{n}^{h}, \mathbf{N}_{n}^{h}\right)$. We state then, $\forall n=1, \ldots, N$

$$
\left\{\begin{array}{c}
E_{i}^{n}=\Phi \tilde{p}_{i, n}-\tilde{p}_{i, n}^{h} \text { and } \mathbf{E}^{n}=\left(E_{i}^{n}\right)_{i} \\
e_{i}^{n}=\psi N_{i, n}-N_{i, n}^{h} \text { and } \mathbf{e}^{n}=\left(e_{i}^{n}\right)_{i} .
\end{array}\right.
$$

Thus, we can write, $\forall \tilde{q}_{h} \in W_{2 h}$

$$
m_{h}^{i}\left(\mathbf{E}^{n+1}, \tilde{q}^{h}\right)+a^{i}\left(\mathbf{e}^{n+1}, \tilde{q}^{h}\right)=0 .
$$

Moreover,

$$
b^{i}\left(v^{h}, \mathbf{E}^{n+1}\right)+\frac{1}{\Delta t} d_{h}^{i}\left(v^{h}, \mathbf{e}^{n+1}-\mathbf{e}^{n}\right)=l^{i, n}\left(v^{h}\right)+b^{i}\left(v^{h}, \Phi \tilde{p}_{n}\right)+\frac{1}{\Delta t} d_{h}^{i}\left(v^{h}, \psi \mathbf{N}_{n+1}-\psi \mathbf{N}_{n}\right) .
$$


Then, with the initial problem (4.4), we obtain

$$
\begin{aligned}
b^{i}\left(v^{h}, \mathbf{E}^{n+1}\right)+\frac{1}{\Delta t} d_{h}^{i}\left(v^{h}, \mathbf{e}^{n+1}-\mathbf{e}^{n}\right)= & -d^{i}\left(v^{h}, \partial_{t} \mathbf{N}_{n}\right)+\frac{1}{\Delta t} d^{i}\left(v^{h}, \psi \mathbf{N}_{n+1}-\psi \mathbf{N}_{n}\right) \\
& -\frac{1}{\Delta t} d_{h}^{i}\left(v^{h}, \psi \mathbf{N}_{n+1}-\psi \mathbf{N}_{n}\right)-d^{i}\left(v^{h}, \psi \mathbf{N}_{n+1}-\psi \mathbf{N}_{n}\right)
\end{aligned}
$$

Considering the solution $\mathbf{N}$ as being enough regular, we state the following estimate

$$
\left\|\sum_{1 \leq l \leq n_{c}} K_{i l} \partial_{t} N_{i, n}-\frac{1}{\Delta t} \sum_{1 \leq l \leq n_{c}} K_{i l}\left(\psi N_{l, n+1}-\psi N_{l, n}\right)\right\|_{0, \Omega} \leq C(\Delta t+h) .
$$

We use the lemma 4.1 to deduce the inequality

$$
\left\|\frac{1}{\Delta t} \sum_{1 \leq l \leq n_{c}} K_{i l} \Pi_{0, h}\left(\psi N_{l, n+1}-\psi N_{l, n}\right)-\left(\psi N_{l, n+1}-\psi N_{l, n}\right)\right\|_{0, \Omega} \leq C \frac{h}{\Delta t} \sum_{1 \leq l \leq n_{c}} K_{i l}\left\|\psi N_{l, n+1}-\psi N_{l, n}\right\|_{0, \Omega},
$$

and so, for a sufficiently regular solution, we prove the existence of a constant $C>0$, such that,

$$
\left\|\frac{1}{\Delta t} \sum_{1 \leq l \leq n_{c}} K_{i l} \Pi_{0, h}\left(\psi N_{l, n+1}-\psi N_{l, n}\right)-\left(\psi N_{l, n+1}-\psi N_{l, n}\right)\right\|_{0, \Omega} \leq C . h .
$$

Then, we state

$$
\begin{aligned}
\epsilon_{i}^{n}=\sum_{1 \leq l \leq n_{c}} K_{i l} \partial_{t} N_{i, n}-\frac{1}{\Delta t} \sum_{1 \leq l \leq n_{c}} K_{i l}\left(\psi N_{l, n+1}\right. & \left.-\psi N_{l, n}\right) \\
& +\frac{1}{\Delta t} \sum_{1 \leq l \leq n_{c}} K_{i l}\left[\Pi_{0, h}\left(\psi N_{l, n+1}-\psi N_{l, n}\right)-\left(\psi N_{l, n+1}-\psi N_{l, n}\right)\right] .
\end{aligned}
$$

According to the previous results, we conclude that

$$
\forall i \in\left\{1 . . n_{c}\right\}, \exists C_{i}>0, \text { such that }\left\|\epsilon_{i}^{n}\right\|_{0, \Omega} \leq C_{i}(\Delta t+h) .
$$

\subsection{Convergence of the numerical scheme}

As mentioned above, we proved that

$$
\begin{aligned}
& \forall 1 \leq i \leq n_{c}, \forall \tilde{q}^{h} \in W_{2 h}, m_{h}^{i}\left(R\left(\mathbf{E}^{n+1}\right), \tilde{q}^{h}\right)+a^{i}\left(\mathbf{e}^{n+1}, \tilde{q}^{h}\right)=0, \\
& \forall 1 \leq i \leq n_{c}, \forall v^{h} \in M_{2 h}, b^{i}\left(v^{h}, \mathbf{E}^{n}\right)+\frac{1}{\Delta t} d_{h}^{i}\left(v^{h}, \mathbf{e}^{n+1}-\mathbf{e}^{n}\right)=\int_{\Omega} \epsilon_{i}^{n} v^{h} \mathrm{~d} x .
\end{aligned}
$$

We are now able to prove the following convergence result:

Theorem 4.3. For a "regular enough" solution $\mathbf{N}=\left(N_{i}\right)_{1 \leq i \leq n_{c}}$,

$$
\forall i \in\left\{1 . . n_{c}\right\}, \exists C_{i}>0 \text {, such that } \max _{n}\left|N_{i, n}^{h}-N_{i, n}\right| \leq C_{i}(\Delta t+h) .
$$

Proof. In (4.5) and (4.6) we chose the following test-functions

$$
v^{h}=\Pi_{0, h}\left(e_{i}^{n+1}-e_{i}^{n}\right), \quad \tilde{q}^{h}=R^{*}\left(e_{i}^{n+1}-e_{i}^{n}\right) .
$$


Then we have,

$$
b^{i}\left(e_{i}^{n+1}-e_{i}^{n}, \mathbf{E}^{n}\right)+\frac{1}{\Delta t} d_{h}^{i}\left(\Pi_{0, h}\left(e_{i}^{n+1}-e_{i}^{n}\right), \mathbf{e}^{n+1}-\mathbf{e}^{n}\right)=\int_{\Omega} \epsilon_{i}^{n} \Pi_{0, h}\left(e_{i}^{n+1}-e_{i}^{n}\right) \mathrm{d} x .
$$

We use the Green's formula to state

$$
\begin{aligned}
b^{i}\left(e_{i}^{n+1}-e_{i}^{n}, \mathbf{E}^{n}\right) & =m_{i}^{h}\left(R\left(\mathbf{E}^{n}\right), R^{*}\left(\mathbf{e}^{n+1}-\mathbf{e}^{n}\right)\right) \\
& =\int_{\Omega} \tilde{\nabla} e_{i}^{n} \cdot R^{*}\left(e_{i}^{n+1}-e_{i}^{n}\right) \mathrm{d} x
\end{aligned}
$$

and thus

$$
\int_{\Omega} \tilde{\nabla} e_{i}^{n} \cdot R^{*}\left(e_{i}^{n+1}-e_{i}^{n}\right) \mathrm{d} x+\frac{1}{\Delta t} d_{h}^{i}\left(\Pi_{0, h}\left(e_{i}^{n+1}-e_{i}^{n}\right), \mathbf{e}^{n+1}-\mathbf{e}^{n}\right)=\int_{\Omega} \epsilon_{i}^{n} \Pi_{0, h}\left(e_{i}^{n+1}-e_{i}^{n}\right) \mathrm{d} x .
$$

We sum over each component in order to use the coercivity assumption on the adsorption tensor to deduce the inequality

$$
\sum_{1 \leq i \leq n_{c}} \int_{\Omega} \tilde{\nabla} e_{i}^{n} \cdot R^{*}\left(e_{i}^{n+1}-e_{i}^{n}\right) \mathrm{d} x+\frac{\alpha}{\Delta t}\left\|\Pi_{0, h}\left(\mathbf{e}^{n+1}-\mathbf{e}^{n}\right)\right\|_{0, \Omega}^{2} \leq \sum_{1 \leq i \leq n_{c}} \int_{\Omega}\left|\epsilon_{i}^{n} \Pi_{0, h}\left(e_{i}^{n+1}-e_{i}^{n}\right)\right| \mathrm{d} x .
$$

Then, considering the definition of the norm $|$.$| and the Young's inequality, we obtain$

$$
\sum_{1 \leq i \leq n_{c}}\left[\left|e_{i}^{n+1}\right|^{2}-\left|e_{i}^{n}\right|^{2}-\left|e_{i}^{n+1}-e_{i}^{n}\right|^{2}\right]+\frac{\alpha}{\Delta t}\left\|\Pi_{0, h}\left(\mathbf{e}^{n+1}-\mathbf{e}^{n}\right)\right\|_{0, \Omega}^{2} \leq \frac{\Delta t}{\alpha} \sum_{1 \leq i \leq n_{c}}\left\|\epsilon_{i}^{n}\right\|_{0, \Omega}^{2} .
$$

Now, obviously,

$$
\exists C_{1, i}>0, \text { such that }\left|e_{i}^{n+1}-e_{i}^{n}\right|^{2} \leq \frac{C_{1, i}}{h^{2}}\left\|\left(e_{i}^{n+1}-e_{i}^{n}\right)\right\|_{0, \Omega}^{2} .
$$

Then, for specific values of the time step $\Delta t$ given by

$$
\Delta t \leq \frac{\alpha h^{2}}{2 n_{c} \max _{i \in\left\{1 . . n_{c}\right\}}\left(C_{i}^{*} C_{1, i}\right)}
$$

(where the constants $\left(C_{i}^{*}\right)$ are given by the Lemma 4.2), we obtain the following inequality

$$
\sum_{1 \leq i \leq n_{c}}\left[\left|e_{i}^{n+1}\right|^{2}-\left|e_{i}^{n}\right|^{2}\right] \leq \frac{\Delta t}{\alpha} \sum_{1 \leq i \leq n_{c}}\left\|\epsilon_{i}^{n}\right\|_{0, \Omega}^{2}
$$

and thus, summing over the time iterations, we have, $\forall i \in\left\{1 . . n_{c}\right\}$

$$
\left|e_{i}^{n+1}\right| \leq C_{i}(\Delta t+h) .
$$




\section{An upscaling method}

We describe here the results obtained with the application of homogenization methods to the equations treated in the previous parts. We try to determine their limit state in order to obtain an accurate modelization of thermodiffusion phenomena in porous media, of which the macroscopic description is still unsufficiently known.

We consider a nonempty bounded open part $\Omega$ of $\mathbb{R}^{3}$ which boundary is regular, and we denote by $Y=\left[0,1{ }^{3}\right.$ the semi-open unity cube of $\mathbb{R}^{3}$. This cell is divided in two parts, the solid part $Y_{s}$ and the free medium $Y_{f}$ such that $Y_{f} \cap \operatorname{Int}(Y)$ is an open part.

We introduce then the medium's porosity $\phi$, defined by

$$
\phi=\frac{\mathcal{L}^{3}-\operatorname{meas}\left(Y \backslash Y_{s}\right)}{\mathcal{L}^{3}-\operatorname{meas}(Y)}=\frac{\operatorname{meas}\left(Y_{f}\right)}{\operatorname{meas}(Y)} .
$$

Let us state $\varepsilon$ a nonnegative real. We can divide $\mathbb{R}^{n}$ in a free part and the substrate in the following manner

$$
E_{f}=\bigcup_{z \in \mathbb{Z}^{n}}\left(z+Y_{f}\right), \quad E_{s}=\bigcup_{z \in \mathbb{Z}^{n}}\left(z+Y_{s}\right) .
$$

It remains to define the fluid flow domain with the expression

$$
\Omega_{f, \varepsilon}=\Omega \cap \varepsilon E_{f}
$$

and the part

$$
C_{\varepsilon}=\operatorname{Int}\left(\bigcup_{\varepsilon(z+Y) \subset \Omega} \varepsilon(z+Y)\right) .
$$

Then we introduce the following notations

$$
\Omega_{f, \varepsilon}^{0}=C_{\varepsilon} \cap \varepsilon E_{f} \quad \Omega_{\varepsilon, s}^{0}=\Omega \backslash \overline{\Omega_{f, \varepsilon}^{0}} .
$$

We classically use the following assumptions on $Y, Y_{f}, Y_{s}, E_{f}, E_{s}$ and their boundaries, these assumptions having already been introduced in [2] and [5]. We assume particularly that this partition is a real one, i.e.

$$
\min \left(\operatorname{meas}\left(Y_{f}\right), \operatorname{meas}\left(Y_{f}\right)\right)>0,
$$

and that the set $Y_{f} \cap \operatorname{int}(Y)$ is an open and connected part* with a Lipschitz boundary. We introduce different notations relative to the functions used in the analysis. We define the notion of extension or again the mean notion. In the following part, for all function $f$ defined on $\Omega_{\varepsilon}$, we denote by $\bar{f}$ its extension by zero on $\Omega$, out of $\Omega_{\varepsilon}$. In the same way, for all function $f$ of $L^{1}(\Omega)$ where $\Omega$ is an open part of $\mathbb{R}^{3}$, we denote by $\tilde{f}$ its mean over this open part, i.e. the real value

$$
\tilde{f}=\frac{1}{\operatorname{meas}(\Omega)} \int_{\Omega} f(x) \mathrm{d} x .
$$

as a rule, the suffix $\sharp$ will denote a periodic function. So, $f \in L_{\sharp}^{2}(Y)$ is equivalent to $f \in L^{2}(Y)$ and $f$ is an Y-periodic function. In the following part, the function $\chi_{\Omega_{f, \varepsilon}}$ refers to characteristic function of the fluid

*In [2], the author gives geometrical representations of situations where these assumptions are not verified, and an illustration of the connectivity notion. 
medium, i.e. the function defined by

$$
\forall x \in \Omega, \quad \chi_{\Omega_{f, \varepsilon}}(x)=\left\{\begin{array}{l}
1 \text { if } x \in \Omega_{f, \varepsilon} \\
0 \text { elsewhere. }
\end{array}\right.
$$

\subsection{From Navier-Stokes to linear Darcy}

The treatment of flow equations has been made using asymptotic developments and will not be detailed here. We used the technique introduced by Diaz in [9], which is based on the particular choice of coefficients in the asymptotic development of the velocity, which allows to obtain the rigorous proof of the transition from the evolutive Navier-Stokes equation to the Darcy's equation. This mathematical transition from a phenomenological law to an empiric one has been studied a lot. A rigorous demonstration of the homogenization of permanent Navier-Stokes equation has been done by Tartar in [24], while the homogenization of "Stokes type" equations has been studied by Antontsev et al. in [5].

\subsection{Homogenization of the heat transfer}

We consider the energy equation in the permanent state, with some boundary conditions at the free mediaporous media interface, the temperature and the fluxes continuity, associated to respective thermal diffusivities.

$$
\left\{\begin{array}{l}
\kappa_{f} \Delta \theta+\mathbf{U} \cdot \nabla \theta=0 \text { in the free medium } \\
\kappa_{s} \Delta \theta=0 \text { in the porous structure. }
\end{array}\right.
$$

This equation is different from the two others in the sense that the thermal field is defined everywhere on the domain and it is not necessary to introduce extensions by zero. The variational formulation associated to this problem is the following one:

$$
\left(E_{\varepsilon}^{\theta}\right)\left\{\begin{array}{l}
\forall v \in H_{0}^{1}(\Omega), \int_{\Omega} \kappa\left(\frac{x}{\varepsilon}\right) \nabla \theta_{\varepsilon} \cdot \nabla v \mathrm{~d} x+\int_{\Omega} \chi_{\Omega_{f, \varepsilon}} \theta_{\varepsilon} \mathbf{U}_{\varepsilon} \cdot \nabla v \mathrm{~d} x=0 \\
\left.\theta_{\varepsilon}\right|_{\partial \Omega}=g
\end{array}\right.
$$

where $g$ is a function in $H^{\frac{1}{2}}(\partial \Omega)$ and $\kappa$ the function defined by

$$
\begin{aligned}
\kappa(.) & =\kappa_{f} \chi\left(Y \backslash \bar{Y}_{s}\right)+\kappa_{s} \chi\left(Y_{s}\right) \\
& =\kappa_{f} \chi\left(Y_{f}\right)+\kappa_{s} \chi\left(Y_{s}\right),
\end{aligned}
$$

with $\kappa_{f}$ and $\kappa_{s}$ the thermal diffusivities of the solid and the fluid media.

Our aim here is to use a process of two scale convergence, in order to dispose of a strong enough convergence on the temperature to introduce it in the mass conservation equation and to conclude. We distinguish in the following argumentation four main parts; first, we deduce with a priori estimates a result of two scale convergence for the unknown $\theta_{\varepsilon}$ (Th. 5.1). Secondly, we multiply the micro state equation by appropriated test-functions in order to obtain a variational formulation at the limit state. An integration part by part allows then to determine the macroscopic problem. A last step consists in eliminating local variables in the macroscopic problem by decoupling this one from a problem posed on an elementary cell (Th. 5.2).

We recall in a first time the following result:

Theorem 5.1. The generalized sequences $\left(\theta_{\varepsilon}\right)_{\varepsilon}$ and $\left(\nabla \theta_{\varepsilon}\right)_{\varepsilon}$ two-scale converge respectively to elements $\theta^{*}(x)$ of $H^{1}(\Omega)$ and $\left(\nabla_{x} \theta^{*}+\nabla_{y} \xi(x, y)\right)$ of $H^{1}(\Omega) \times L^{2}\left[\Omega ; H_{\sharp}^{1}(Y) \backslash \mathbb{R}\right]$. 
The proof of this theorem has already been done in the case of perforated media by Allaire in [1]. Now, we are able to determine the homogenized problem verified by the limit state $\theta^{*}$ :

Theorem 5.2. $\theta^{*}$ is the unique solution in $H^{1}(\Omega)$ of the following homogenized problem

$$
\left(E^{\theta^{*}}\right)\left\{\begin{array}{l}
\forall v \in H_{0}^{1}(\Omega), \int_{\Omega} \tilde{\Lambda} \nabla \theta^{*} \cdot \nabla v \mathrm{~d} x+\int_{\Omega} \phi \theta^{*} \mathbf{U} \cdot \nabla v \mathrm{~d} x=0 \\
\left.\theta^{*}\right|_{\partial \Omega}=g
\end{array}\right.
$$

where $\tilde{\Lambda}$ is the elliptical tensor given by

$$
\begin{aligned}
\Lambda_{k l} & =\int_{Y} \kappa\left(\nabla_{y} \sigma_{k}+\vec{e}_{k}\right) \cdot \vec{e}_{l} \mathrm{~d} y \\
& =\int_{Y_{f}} \kappa_{f}\left(\nabla_{y} \sigma_{k}+\vec{e}_{k}\right) \cdot \vec{e}_{l} \mathrm{~d} y+\int_{Y_{s}} \kappa_{s}\left(\nabla_{y} \sigma_{k}+\vec{e}_{k}\right) \cdot \vec{e}_{l} \mathrm{~d} y
\end{aligned}
$$

and $\left(\sigma_{k}\right)$ is a family of solutions of the following problem

$$
\left(E_{\text {cell }}^{\theta}\right)\left\{\begin{array}{l}
\sigma_{k} \in H_{\sharp}^{1}(Y) \\
\operatorname{div}_{y}\left(\kappa_{f}\left[\nabla_{y} \sigma_{k}+\vec{e}_{k}\right]\right)=0 \operatorname{in} Y_{f} \\
\operatorname{div}_{y}\left(\kappa_{s}\left[\nabla_{y} \sigma_{k}+\vec{e}_{k}\right]\right)=0 \operatorname{in} Y_{s} \\
{\left[\kappa_{f}\left(\nabla_{y} \sigma_{k}+\vec{e}_{k}\right)-\kappa_{s}\left(\nabla_{y} \sigma_{k}+\vec{e}_{k}\right)\right] \cdot \vec{n}=0 \text { on } \partial Y_{f} \backslash \partial Y .}
\end{array}\right.
$$

Proof. We rewrite the energy equation in free medium (5.3) and multiplying this one by the test function $\psi(x)+\psi_{1}(x, y)$ where $\psi \in D(\Omega)$ and $\psi_{1} \in D\left[\Omega ; \mathcal{C}_{\sharp}^{\infty}(Y)\right]$, we obtain, with a Green's formula,

$$
\begin{aligned}
-\int_{\Omega} \int_{Y} \kappa\left(\frac{x}{\varepsilon}\right) \nabla \theta_{\varepsilon} \cdot \nabla\left(\psi(x)+\varepsilon \psi_{1}\left(x, \frac{x}{\varepsilon}\right)\right) \mathrm{d} x \mathrm{~d} y & -\int_{\Omega} \int_{Y} \chi_{\Omega_{f, \varepsilon}} \theta_{\varepsilon} \mathbf{U}_{\varepsilon} \cdot \nabla\left(\psi(x)+\varepsilon \psi_{1}\left(x, \frac{x}{\varepsilon}\right)\right) \mathrm{d} x \mathrm{~d} y \\
& =\int_{\Omega} \int_{Y} f_{\varepsilon}\left(\psi(x)+\varepsilon \psi_{1}\left(x, \frac{x}{\varepsilon}\right)\right) \mathrm{d} x \mathrm{~d} y .
\end{aligned}
$$

Noticing that

$$
\nabla\left(\psi(x)+\varepsilon \psi_{1}(x, y)\right)=\nabla_{x} \psi(x)+\nabla_{y} \psi_{1}(x, y)+\varepsilon \nabla_{x} \psi_{1}(x, y),
$$

we obtain, passing to the limit when $\varepsilon \rightarrow 0$,

$$
\begin{aligned}
-\int_{\Omega} \int_{Y} \kappa(y)\left[\nabla_{x} \theta^{*}+\nabla_{y} \xi(x, y)\right] \cdot\left[\nabla_{x} \psi(x)\right. & \left.+\nabla_{y} \psi_{1}(x, y)\right] \mathrm{d} x \mathrm{~d} y \\
& -\int_{\Omega} \int_{Y} \chi_{\Omega_{f}} \phi \theta^{*} \mathbf{U} \cdot\left[\nabla_{x} \psi(x)+\nabla_{y} \psi_{1}(x, y)\right] \mathrm{d} x \mathrm{~d} y=\int_{\Omega} \int_{Y} f \psi(x) \mathrm{d} x \mathrm{~d} y
\end{aligned}
$$

for all $\left(\psi, \psi_{1}\right) \in D(\Omega) \times D\left[\Omega ; \mathcal{C}_{\sharp}^{\infty}(Y)\right]$ and thus, by density, for all $\left(\psi, \psi_{1}\right) \in H_{0}^{1}(\Omega) \times L^{2}\left[\Omega ; H_{\sharp}^{1}(Y) / \mathbb{R}\right]$. We will first have defined the function $f=\operatorname{div}\left(\kappa(x) \nabla \hat{g}+\phi \chi_{\Omega_{f}} \hat{g} \mathbf{U}\right)$. The problem can then be interpreted by

$$
\left\{\begin{array}{l}
\operatorname{div}_{y}\left(\kappa(y)\left(\nabla \theta^{*}+\nabla_{y} \xi(x, y)\right)\right)=0 \text { in } \Omega \times Y \\
\operatorname{div}_{x}\left(\int_{Y} \kappa(y)\left(\nabla \theta^{*}+\nabla_{y} \xi(x, y)\right) \mathrm{d} y+\chi_{\Omega_{f}} \phi \theta^{*} \mathbf{U}\right)=0 \text { in } \Omega \times Y \\
\left.\theta^{*}\right|_{\partial \Omega}=g
\end{array}\right.
$$


This problem having a unique solution (the proof of this result is obvious as soon as one has observed that $\left\|\nabla_{x} \theta^{*}+\nabla_{y} \xi\right\|_{L^{2}(\Omega \times Y)^{n}}$ is a norm for the space $\left.H_{0}^{1}(\Omega) \times L^{2}\left[\Omega ; H_{\sharp}^{1}(Y) \backslash \mathbb{R}\right]\right)$, we can conclude that the entire sequence $\left(\theta_{\varepsilon}\right)$ (resp. $\nabla \theta_{\varepsilon}$ ) converges to $\theta^{*}(x)$ (resp. $\left(\nabla \theta^{*}(x)+\nabla_{y} \xi(x, y)\right.$ ). The first equation represents a local problem (on an elementary cell) allowing to determine the function $\xi$. The second one is the homogenized energy equation. In order to have a better characterization of the homogenized problem, one can eliminate the local variable $y$ from this formulation. This is done in the sequel of this proof.

Let us consider the solutions $\sigma_{k}(x, y)$ of the problem $\left(E_{\text {cell }}^{\theta}\right)$, and the function $\xi(x, y)$ defined by the relation

$$
\xi(x, y)=\sum_{k=1}^{3} \frac{\partial \theta^{*}}{\partial x_{k}} \sigma_{k}(x, y)
$$

A simple calculus allows to verify that this function is a solution of the equation on the elementary cell. It remains now to determine the macroscopic form of the equation

$$
\operatorname{div}_{x}\left(\int_{Y} \kappa(y)\left(\nabla \theta^{*}+\nabla_{y} \xi(x, y)\right) \mathrm{d} y+\chi_{\Omega_{f}} \phi \theta^{*} \mathbf{U}\right)=0 \text { in } \Omega \times Y .
$$

Considering (5.8), we easily establish that

$$
\int_{Y} \kappa(y)\left[\nabla \theta^{*}+\nabla_{y} \xi(x, y)\right] \mathrm{d} y=\sum_{k=1}^{3} \frac{\partial \theta^{*}}{\partial x_{k}}\left(\int_{Y} \kappa(y)\left[\vec{e}_{k}+\nabla_{y} \sigma_{k}(x, y)\right] \mathrm{d} y\right)
$$

Having defined the tensor $\tilde{\Lambda}$ by

$$
\Lambda_{k l}=\int_{Y} \kappa(y)\left[\vec{e}_{k}+\nabla_{y} \sigma_{k}(x, y)\right] \cdot \vec{e}_{l} \mathrm{~d} y
$$

it comes, with the help of Green's formula,

$$
\left(E^{\theta^{*}}\right)\left\{\begin{array}{l}
\forall v \in H_{0}^{1}(\Omega), \int_{\Omega} \tilde{\Lambda} \nabla \theta^{*} \cdot \nabla v \mathrm{~d} x+\int_{\Omega} \phi \theta^{*} \mathbf{U} \cdot \nabla v \mathrm{~d} x=0 \\
\left.\theta^{*}\right|_{\partial \Omega}=g .
\end{array}\right.
$$

The homogenized problem has been entirely determined. We remark that it is a problem very similar to the one posed in a free medium. The study of such a problem is not necessary as soon as the tensor introduced has properties (symmetry, pseudo-ellipticity) that allow to conclude immediately, using the study made in the first part, about existence and uniqueness of a solution.

The complete determination of the thermal field in the porous medium via the homogenized equation requires the resolution of the problem $\left(E_{\text {cell }}^{\theta}\right)$ and the knowledge of the functions $\left(\sigma_{k}\right)$ and more precisely the estimation of energies relative to these functions (the term $\Lambda_{k l}$ ), less expensive in terms of calculus.

Remark 5.1. In the case of constant thermal diffusivities in free and porous media, the problem $\left(E_{\text {cell }}^{\theta}\right)$ is equivalent to

$$
\left\{\begin{array}{l}
\sigma_{k} \in H_{\sharp}^{1}(Y) \\
\Delta_{y} \sigma_{k}=0 \operatorname{in} Y_{f}, \Delta_{y} \sigma_{k}=0 \operatorname{in} Y_{s}, \\
{\left[\kappa_{f}\left(\nabla_{y} \sigma_{k}+\vec{e}_{k}\right)-\kappa_{s}\left(\nabla_{y} \sigma_{k}+\vec{e}_{k}\right)\right] \cdot \vec{n}=0 \text { on } \partial Y_{f} \backslash \partial Y .}
\end{array}\right.
$$




\subsection{Soret effect equations}

In this part, we are interested in the homogenization of mass conservation equations of each component of the mixture. The sorption effects are not considered here as they will be treated in a next part. This model is mainly different from the energy one as the quantities $N_{i, \varepsilon}$ are defined on a domain $\Omega_{\varepsilon, f}$ depending on the parameter $\varepsilon$. This difficulty is overcame by introducing an extension operator.

We consider the convecto-diffusive equations with Soret effect in $\Omega_{f, \varepsilon}$

$$
\forall i \in\{1 . . n\}, \partial_{t} N_{i, \varepsilon}+\mathbf{U}_{\varepsilon} \cdot \nabla N_{i, \varepsilon}-\sum_{j=1}^{n} D_{i j} \Delta N_{j, \varepsilon}-S_{t}^{i} \operatorname{div}\left(N_{i, \varepsilon}\left(1-N_{i, \varepsilon}\right) \nabla \theta_{\varepsilon}\right)=0
$$

associated to initial and boundary conditions

$$
\left\{\begin{array}{l}
\sum_{j=1}^{n} D_{i j} \frac{\partial N_{j, \varepsilon}}{\partial n}+S_{t}^{i} N_{i, \varepsilon}\left(1-N_{i, \varepsilon}\right) \frac{\partial \theta_{\varepsilon}}{\partial n}=0 \\
N_{i, \varepsilon}(x, 0)=N_{i}^{0}
\end{array}\right.
$$

The variational formulation associated to this problem is the following one:

$$
\begin{aligned}
& \int_{\Omega} \chi_{\Omega_{f, \varepsilon}} \partial_{t} N_{i, \varepsilon} v \mathrm{~d} x+\int_{\Omega} \chi_{\Omega_{f, \varepsilon}} N_{i, \varepsilon} \mathbf{U}_{\varepsilon} \cdot \nabla v \mathrm{~d} x \\
& -\sum_{j} D_{i j} \int_{\Omega} \chi_{\Omega_{f, \varepsilon}} \nabla N_{j, \varepsilon} \cdot \nabla v \mathrm{~d} x-\int_{\Omega} \chi_{\Omega_{f, \varepsilon}} S_{t}^{i} N_{i, \varepsilon}\left(1-N_{i, \varepsilon}\right) \nabla \theta_{\varepsilon} \cdot \nabla v \mathrm{~d} x=0
\end{aligned}
$$

\subsubsection{Behaviour of $\bar{N}_{i, \varepsilon}$}

We have seen in the part relative to the mathematical analysis of Soret effect equations (cf. Prop. 1.1) that the unknowns $N_{i, \varepsilon}$ are bounded in $H^{1}\left(Q_{\varepsilon}\right)$, independently of $\varepsilon$. The method consists in searching an extension operator on $H^{1}(Q)$ for the unknowns that allows to conserve such a property. Thus, we introduce the extension operator $P_{\varepsilon}$

$$
P_{\varepsilon}: \mid \begin{aligned}
& H^{1}\left(Q_{\varepsilon}\right) \longrightarrow H^{1}(Q) \\
& N_{i, \varepsilon} \longmapsto P_{\varepsilon}\left(N_{i, \varepsilon}\right)=\bar{N}_{i, \varepsilon}
\end{aligned}
$$

which is continue, with a continuity constant $C_{p}$ independent of $\varepsilon$. This operator, already introduced by Diaz in [9] allows to conserve a priori estimates, independently of $\varepsilon$. As a direct consequence, we have

$$
\left\|\bar{N}_{i, \varepsilon}\right\|_{1, Q} \leq C_{p}\left\|N_{i, \varepsilon}\right\|_{1, Q_{\varepsilon}} \leq C^{\prime}
$$

$C^{\prime}$ being independent of $\varepsilon$. Since $Q$ is a bounded lipschitzian part, we can apply the Rellich Kondrachoff theorem (cf. [18] or again [19])which ensure us of the compacity of $H^{1}(Q)$ in $L^{2}(Q)$. The inequality (5.12) allows to prove the existence of an extracted subsequence of $\bar{N}_{i, \varepsilon}$ that converges weakly in $H^{1}(Q)$. The injection of $H^{1}(Q)$ in $L^{2}(Q)$ being compact, there exists an extracted subsequence -again denoted by $\left(\bar{N}_{i, \varepsilon}\right)$ - which converges strongly in $L^{2}(Q)$ and almost everywhere in $Q$ to a limit $N_{i}$.

Then we have the following convergence results:

$$
\begin{aligned}
\chi_{\Omega_{f, \varepsilon}} & \rightarrow \phi \chi_{\Omega} \text { in } L^{\infty} \text { weakly }-* \\
\nabla \theta_{\varepsilon} & \rightarrow \nabla \theta^{*} \text { in }\left(L^{2}(\Omega)\right)^{3} \text { weakly } \\
N_{i, \varepsilon} & \rightarrow N_{i} \text { in } H^{1}(Q) \text { weakly } \\
N_{i, \varepsilon} & \longrightarrow N_{i} \text { in } L^{2}(Q) \text { and a.e. in Q. }
\end{aligned}
$$


In the sequel, we detail the passing to the limits in each term of the equation.

Theorem 5.3. The sequence $\bar{N}_{i, \varepsilon}$ converges to the solution of a problem associated with the variationnal formulation

$$
\begin{aligned}
& \int_{\Omega} \phi \partial_{t} N_{i} v \mathrm{~d} x+\int_{\Omega} \phi N_{i} \mathbf{U} \cdot \nabla v \mathrm{~d} x \\
& -\sum_{j} D_{i j} \int_{\Omega}\left[\tilde{\Upsilon} \nabla N_{j}\right] \cdot \nabla v \mathrm{~d} x-\int_{\Omega} N_{i}\left(1-N_{i}\right)\left[\tilde{\Sigma}_{i} \nabla \theta^{*}\right] \cdot \nabla v \mathrm{~d} x=0
\end{aligned}
$$

where $\tilde{\Upsilon}_{i}$ and $\tilde{\Sigma}_{i}$ are the tensors defined by

$$
\begin{aligned}
\left(\tilde{\Sigma}_{i}\right)_{k l} & =S_{t}^{i}(\tilde{\Upsilon})_{k l} \\
& =\frac{S_{t}^{i}}{\mathcal{L}^{3}-\operatorname{meas}(Y)} \int_{Y_{f}}\left(\nabla_{y} \omega_{k}+\overrightarrow{e_{k}}\right)\left(\nabla_{y} \omega_{l}+\overrightarrow{e_{l}}\right) \mathrm{d} y
\end{aligned}
$$

with $\left(\omega_{k}\right)_{k=1,2}$ is a family of functions, solutions of problems on the elementary cell $Y$

$$
\left\{\begin{array}{l}
\omega_{k} \in H_{\sharp}^{1}(Y) \\
-\operatorname{div}_{y}\left(\nabla_{y} \omega_{k}+\vec{e}_{k}\right)=0 \text { in } Y_{f} \\
\left(\nabla_{y} \omega_{k}+\vec{e}_{k}\right) \cdot \vec{n}=0 \text { on } \partial Y_{f} \backslash \partial Y .
\end{array}\right.
$$

Proof. The proof of the convergence of diffusive and convective terms is similar to the one done for the energy equation problem. We treat now the passing to the limit in the nonlinear term of Soret effect $\int_{\Omega} \chi_{\Omega_{\varepsilon}} S_{t}^{i} N_{\varepsilon, i}(1-$ $\left.N_{\varepsilon, i}\right) \nabla \theta_{\varepsilon} \cdot \nabla v \mathrm{~d} x$. With this aim in view, we use the convergence results given in (5.13) and (5.14) which allow to establish that

$$
\chi_{\Omega_{\varepsilon}} \nabla \theta_{\varepsilon} \rightarrow \tilde{\Upsilon} \nabla \theta^{*} \text { in }\left(L^{2}(\Omega)\right)^{3} \text { weakly },
$$

the tensor $\tilde{\Upsilon}$ being calculable with auxiliary functions constructed on $Y_{f}$. Then we use the convergence result (5.16) in order to prove the existence of a subsequence (again denoted by $N_{\varepsilon, i}$ ) that converges strongly in $L^{2}(Q)$ to a limit $N_{i}$. So the sequence $N_{\varepsilon, i}\left(1-N_{\varepsilon, i}\right) \nabla v$ converges strongly to a limit $N_{i}\left(1-N_{i}\right) \nabla v$ in $\left(L^{2}(Q)\right)^{3}$, and then

$$
\lim _{\varepsilon \rightarrow 0} \int_{Q} \chi_{\Omega_{\varepsilon}} S_{t}^{i} N_{\varepsilon, i}\left(1-N_{\varepsilon, i}\right) \nabla \theta_{\varepsilon} \cdot \nabla v \mathrm{~d} x=\int_{Q} N_{i}\left(1-N_{i}\right)\left[\tilde{\Sigma}_{i} \nabla \theta^{\star}\right] \cdot \nabla v \mathrm{~d} x
$$

$\tilde{\Sigma}$ being the tensor defined by $\tilde{\Sigma}=S_{t}^{i} \tilde{\Upsilon}$.

Remark 5.2. The tensor $\tilde{\Upsilon}$ can also be rewritten, using problems (5.20) as follows:

$$
\begin{aligned}
\Upsilon_{k l} & =\frac{1}{\mathcal{L}^{3}-\operatorname{meas}(Y)}\left\{\int_{Y_{f}} \frac{\partial \omega_{k}}{\partial y_{l}} \mathrm{~d} y+\operatorname{mes}\left(Y_{f}\right) \delta_{k l}\right\} \\
& =\phi \delta_{k l}+\frac{1}{\mathcal{L}^{3}-\operatorname{meas}(Y)} \int_{Y_{f}} \frac{\partial \omega_{k}}{\partial y_{l}} \mathrm{~d} y .
\end{aligned}
$$

We recognize a formulation similar to the one of $\tilde{\Lambda}$ in (5.6), having on the diagonal the porosity (modulo the diffusive coefficients). The symmetry of the tensor $\tilde{\Upsilon}$ is no more obvious, but implicitly given by elementary problems. 
Remark 5.3. One can notice the importance in this process of the existence of the operator $P_{\varepsilon}$. The fact that the continuity constant $C_{p}$ of $P_{\varepsilon}$ does not depend on $\varepsilon$ is of capital importance in the proof. The main difficulty in the proof of the convergence remains in the fact that quantities $N_{i, \varepsilon}$ are defined on a part $\Omega_{\varepsilon, f}$; as Allaire recalls in [1], two methods can be used to overcome this problem. Other techniques can be used, as the proof of the compacity of the injection of $H^{1}\left(\Omega_{\varepsilon, f}\right)$ in $L^{2}\left(\Omega_{\varepsilon, f}\right)$, which is uniform in $\varepsilon$ (this is an adapted version of Rellich theorem to the perforated media).

\subsection{Upscaling in sorption effects}

We are now interested in the contamination of aquifer layers by adsorption of pollutant. The modelization of such phenomena requires the knowledge of adsorption isotherms of each specie (function linking the adsorbed concentration by surface unity to the free one). These isotherms can be represented by simple functions which main parameters are temperature and the nature of the solid. We consider the adsorption in a general way and perform the analysis for two extreme cases: the irreversible case (the solid fixes definitively the pollutant) and the reversible one (all the matter adsorbed is rejected in the fluid phase). The application of homogenization processes to such phenomena has been already done by Hornung in [15] or Hornung and Jäger in [16], but the proof of the convergence had not been established.

\subsubsection{Statement of the problem}

In this part, we are interested in adsorption phenomena occurring on the porous surface. The repartition of the species into the fluid is modelized by a classic "convecto-diffusive" equation

$$
\partial_{t} N_{\varepsilon}+\operatorname{div}\left(N_{\varepsilon} \mathbf{U}_{\varepsilon}-D \nabla N_{\varepsilon}\right)=0 \text { in } \Omega_{f, \varepsilon}
$$

The adsorption effects are translated by a boundary condition on the fluid-solid interface of the type

$$
-D \frac{\partial N_{\varepsilon}}{\partial n}=\varepsilon \lambda\left(\frac{x}{\varepsilon}\right) \varphi\left(N_{\varepsilon}\right) \text { on } \Gamma_{\varepsilon}
$$

where $\lambda$ is an element of $L_{\sharp}^{\infty}(\Gamma)$ and $D$ the diffusion coefficient of the component in the fluid. The initial and the complete boundary conditions remain similar to the ones considered in the first part. Thus, with a Green's formula, and for a test function $v$ "regular enough" defined on $\bar{Q}$, we obtain the variational formulation

$$
\int_{\Omega_{f, \varepsilon}} \partial_{t} N_{\varepsilon} v \mathrm{~d} x+\int_{\Omega_{f, \varepsilon}} N_{\varepsilon} \mathbf{U}_{\varepsilon} \cdot \nabla v \mathrm{~d} x+D \int_{\Omega_{f, \varepsilon}} \nabla N_{\varepsilon} \cdot \nabla v \mathrm{~d} x-D \int_{\Gamma_{\varepsilon}} v \frac{\partial N_{\varepsilon}}{\partial n} \mathrm{~d} \sigma_{\varepsilon}=0 \text { a.e. in } t .
$$

Then we use the inequality (5.25) in order to obtain the formulation with a sink term

$$
\int_{\Omega_{f, \varepsilon}} \partial_{t} N_{\varepsilon} v \mathrm{~d} x+\int_{\Omega_{f, \varepsilon}} N_{\varepsilon} \mathbf{U}_{\varepsilon} \cdot \nabla v \mathrm{~d} x+D \int_{\Omega_{f, \varepsilon}} \nabla N_{\varepsilon} \cdot \nabla v \mathrm{~d} x=-\varepsilon \int_{\Gamma_{\varepsilon}} \lambda\left(\frac{x}{\varepsilon}\right) \varphi\left(N_{\varepsilon}\right) v \mathrm{~d} \sigma_{\varepsilon}
$$

for "regular enough" functions $v$ defined on $\bar{Q}$. One can easily verify that the problem composed of equations (5.27) and (5.25) admits a unique solution (evolutive parabolic problem with a non linear sink term).

\subsubsection{Irreversible case: The Langmuir isotherm}

We are interested here in the case of full irreversible adsorption, given by the Langmuir's model, one of the most classically used isotherm. The flux at the interface is then described by

$$
\varphi: r \in \mathbb{R} \longmapsto \varphi(r)=\left(\frac{\alpha r}{1+\beta r}-N_{\mathrm{sat}}\right)^{+}
$$

$N_{\text {sat }}$ being a saturation value. 
Proposition 5.1. We have the following estimates:

$$
\begin{array}{ll}
\exists C_{1}>0, & \left\|N_{\varepsilon}\right\|_{H^{1}\left(Q_{\varepsilon}\right)} \leq C_{1} \\
\exists C_{2}>0, & \left\|\varphi\left(N_{\varepsilon}\right)\right\|_{H^{1}\left(Q_{\varepsilon}\right)} \leq C_{2} .
\end{array}
$$

The function $\varphi$ being lipschitzian and vanishing at $r=0$, with a Lipschitz constant $\operatorname{Lip}(\varphi)$, we get that $\varphi\left(N_{\varepsilon}\right)$ is bounded in $H^{1}\left(Q_{\varepsilon}\right)$ and

$$
\left\|\varphi\left(N_{\varepsilon}\right)\right\|_{H^{1}\left(Q_{\varepsilon}\right)} \leq \operatorname{Lip}(\varphi)\left\|N_{\varepsilon}\right\|_{H^{1}\left(Q_{\varepsilon}\right)} .
$$

Proposition 5.2 (Langmuir's isotherm). The generalized sequence $\left(N_{\varepsilon}\right)_{\varepsilon}$ converges to an element $N$, solution of the equation

$$
\phi \partial_{t} N+\operatorname{div}(\phi N \mathbf{U}-D \tilde{\Upsilon} \nabla N)+\left[\int_{\Gamma} \lambda(y) \mathrm{d} \sigma(y)\right] \varphi(N)=0 \text { in } Q .
$$

Proof. Considering the variational formulation verified by $N_{\varepsilon}$, and integrating the inequality $(5.27)$ on $[0, T]$, we have

$$
\begin{aligned}
\int_{0}^{T} \int_{\Omega_{f, \varepsilon}} \partial_{t} N_{\varepsilon} v \mathrm{~d} x \mathrm{~d} t & +\int_{0}^{T} \int_{\Omega_{f, \varepsilon}} N_{\varepsilon} \mathbf{U}_{\varepsilon} \cdot \nabla v \mathrm{~d} x \mathrm{~d} t \\
& +D \int_{0}^{T} \int_{\Omega_{f, \varepsilon}} \nabla N_{\varepsilon} \cdot \nabla v \mathrm{~d} x \mathrm{~d} t=-\varepsilon \int_{0}^{T} \int_{\Gamma_{\varepsilon}} \lambda\left(\frac{x}{\varepsilon}\right) \varphi\left(N_{\varepsilon}\right) v \mathrm{~d} \sigma_{\varepsilon} \mathrm{d} t .
\end{aligned}
$$

The convergence of convective, diffusive and evolutive terms has already been proved in the previous parts. In a first time we use a suitable extension $\tilde{N}_{\varepsilon}$ of the unknowns $N_{\varepsilon}$ in order to obtain a constant $C>0$, independent of $\varepsilon$, such that

$$
\left\|\tilde{N}_{\varepsilon}\right\|_{H^{1}\left(Q_{\varepsilon}\right)} \leq C\left\|N_{\varepsilon}\right\|_{H^{1}\left(Q_{\varepsilon}\right)}
$$

The tricky point consists in passing to the limit in the term $\varepsilon \int_{\Gamma_{\varepsilon}} \lambda\left(\frac{x}{\varepsilon}\right) \varphi\left(N_{\varepsilon}\right) v \mathrm{~d} \sigma_{\varepsilon}$. With this aim in view, we use results of two scale convergence for the expressions on the boundaries. These results, introduced by Allaire, Damlamian and Hornung in [3], are the generalization to the boundaries of the two scale convergence notion introduced in [2] and have been applied to diffusive equations with Fourier type boundary conditions. In the following, we will denote by

$$
u_{\varepsilon} \stackrel{2-\text { scale }}{\Gamma} u_{0}
$$

this type of convergence.

The adaptation of this notion to our model do not give any difficulty with the help of the proposition (5.1). Thus, taking for test function $v=\varphi\left(N_{\varepsilon}\right)$ in the variational formulation (5.27), we easily obtain the inequality

$$
\varepsilon \int_{0}^{T} \int_{\Gamma_{\varepsilon}}\left|\lambda\left(\frac{x}{\varepsilon}\right) \varphi(x)\right|^{2} \mathrm{~d} \sigma_{\varepsilon}(x) \leq C .
$$

Therefore, as mentioned in [3], there exists a function $\varphi(x, y) \in L^{2}\left(\Omega ; L^{2}(\Gamma)\right)$ such that

$$
\varphi_{\varepsilon} \stackrel{2-\text { scale }}{\Gamma} \varphi
$$


We have the following convergence properties:

$$
\varepsilon^{\prime} \int_{0}^{T} \int_{\Gamma_{\varepsilon^{\prime}}} \lambda\left(\frac{x}{\varepsilon^{\prime}}\right) \varphi\left(N_{\varepsilon^{\prime}}\right) \phi\left(t, x, \frac{x}{\varepsilon^{\prime}}\right) \mathrm{d} \sigma_{\varepsilon^{\prime}}(x) \mathrm{d} t \stackrel{\varepsilon^{\prime} \rightarrow 0}{\longrightarrow} \int_{Q} \int_{\Gamma} \lambda(y) \varphi(N) \phi(t, x, y) \mathrm{d} \sigma(y) \mathrm{d} x \mathrm{~d} t
$$

for each continuous function $\phi(x, y) \in \mathcal{C}\left[\bar{\Omega} ; \mathcal{C}_{\sharp}(Y)\right]$. Moreover, with the compacity of the injection from $H^{1}(Q)$ in $L^{2}(Q)$, it comes

$$
\begin{aligned}
& \tilde{N}_{\varepsilon^{\prime}} \rightarrow N \text { in } H^{1}(Q) \text { weakly } \\
& \tilde{N}_{\varepsilon^{\prime}} \rightarrow N \text { in } L^{2}(Q) \text { strongly }
\end{aligned}
$$

Then we have

$$
\varphi\left(\tilde{N}_{\varepsilon^{\prime}}\right) \longrightarrow \varphi(N) \text { in } L^{2}(Q) \text { strongly. }
$$

Remark 5.4. In the case of a constant exchange coefficient $\lambda($.$) , the expression of the nonlinear sink term$ introduced in the macroscopic conservative equation (5.32) becomes

$$
-\lambda \operatorname{meas}(\Gamma) \gamma\left(\frac{\alpha N}{1+\beta N}-N_{\mathrm{sat}}\right)^{+}
$$

The previous proof remains true for all lischitzian function $\varphi$, which is nondecreasing and which vanishes at 0 , that allows to consider a wide set of natural behaviours. In the next section, we present another type of behaviour which corresponds to the case of reversible adsorption.

\subsubsection{The reversible case: The Freundlich isotherm}

We consider here the reversible case which can be modelized by the Freundlich isotherm. This isotherm corresponds to the the function

$$
\varphi: r \in \mathbb{R} \longmapsto \varphi(r)=r^{p}(0<p<1)
$$

which is translated by a condition on the fluid-solid interface

$$
-\frac{\partial N_{\varepsilon}}{\partial n}=\varepsilon \lambda\left|N_{\varepsilon}-N_{\mathrm{sat}}\right|^{p-1}\left(N_{\varepsilon}-N_{\mathrm{sat}}\right) \text { on } \Gamma_{\varepsilon}
$$

Proposition 5.3 (Freundlich isotherm). The macroscopic conservative equation is the following one:

$$
\phi \partial_{t} N+\operatorname{div}(\phi N \mathbf{U}-D \tilde{\Upsilon} \nabla N)=-\lambda \operatorname{meas}(\Gamma)\left|N-N_{\text {sat }}\right|^{p-1}\left(N-N_{\text {sat }}\right) \text { in } Q .
$$

The proof of such a result has been established by Diaz in [9] in the case of the homogenization of chemical reactions between fluid and solid phases at the interface.

\section{Numerical Simulations}

\subsection{The pore scale}

We present here the first simulations obtained using the numerical scheme described in part 4 . These computations have been made on a planar rectangular domain which modelizes a capillary in quasi-linear thermal field. 
TABLE 1. Macroscopic coefficients $\left(\kappa_{f}=1.3 \times 10^{-7} \mathrm{~m}^{2} \cdot \mathrm{s}^{-1}, \kappa_{s}=1.3 \times 10^{-7} \mathrm{~m}^{2} \cdot \mathrm{s}^{-1}, D=\right.$ $\left.2.75 \times 10^{-7} \mathrm{~m}^{2} \cdot \mathrm{s}^{-1}, S_{t}^{*}=4.92 \times 10^{-3}\right)$.

\begin{tabular}{ccc}
\hline Tensor $\backslash$ Cell & Cell 1 & Cell 2 \\
\hline$\tilde{\Lambda}$ & $\left(\begin{array}{cc}3.49 \times 10^{-7} & 0 \\
0 & 3.48 \times 10^{-7}\end{array}\right)$ & $\left(\begin{array}{cc}2.67 \times 10^{-7} & 0 \\
0 & 2.66 \times 10^{-7}\end{array}\right)$ \\
$\tilde{\Sigma}$ & $2.77 \times 10^{-3} I_{2}$ & \\
& & $\left(\begin{array}{cc}1.313 \times 10^{-3} & 0 \\
0 & 1.314 \times 10^{-3}\end{array}\right)$ \\
$\tilde{\Upsilon}$ & $\left(\begin{array}{cc}2.67 \times 10^{-1} & 0 \\
0 & 2.671 \times 10^{-1}\end{array}\right)$ \\
\hline
\end{tabular}

The main parameters used here are the dimensionless Soret coefficients $S_{t}^{i *}=S_{t}^{i} \Delta \theta\left(1-N_{i}(x=0)\right)$, the Peclet numbers $P e^{i}=\frac{\|\mathbf{U}\| L}{D_{i i}}$, the adsorption isotherms of each component and the data relative to the system such as the applied temperature difference $\Delta \theta$ and the velocity profile $U$. The adsorption isotherm chosen for the simulations is the Langmuir isotherm, defined by

$$
\forall i \in\{1 . . n\}, \forall x \in \Omega, \forall y=\left(y_{i}\right)_{1 \leq i \leq n_{c}} \in\{0 . .1\}^{n_{c}}, \quad k^{i}(x, y)=\frac{K_{i}(x) y_{i}}{\phi+\sum_{1 \leq j \leq n_{c}} K_{j}(x) y_{j}}
$$

where $\phi$ denotes the medium porosity and $\left(K_{i}\right)_{1 \leq i \leq n_{c}}$ the Langmuir equilibrium constants family. One can easily show that assumptions $(\mathcal{H})$ hold for the diffusive and adsorption tensors. This isotherm is one of the most simple and most often used functions for the modelization of the adsorption processes, and, if it is well known that it is not the more precise, it remains a good function for the tests, in order to better understand the mechanism that governs the system. In Figure 2(a), we show that the adsorption process and more precisely its speed is modified by the thermal profile (via the Langmuir coefficients): in a first time the elution occurs normally (first visualization) before that the fluid reaches the non isothermal area where the front part is accelerated, while the back part is not disturbed. That's why we observe a deformation of the initial velocity profile (which is more stretched) before reaching the capillary outlet (third visualization), while the desorption process occurs, slowed down by viscous effects. These effects can be better visualized on the isovalue graph for the concentrations of the first component (Fig. 2(b)).

\subsection{Upscaling}

A second set of simulations has been made in order to compute precisely the macroscopic diffusion (and thermodiffusion) tensors determined in part 5 . An unstructured mesh for the unit cell has been constructed. The resolution of elementary problems (5.7) and (5.20) has been done using a finite volume code. The type of elementary cell chosen for these simulations is a stack of balls of "cell centered" type (cell 1 porosity $\phi_{1}=0.72$ ) or "cubic face centered type" (cell 2 , porosity $\phi_{2}=0.43$ ). Some solutions of the auxiliary problems can be visualized in Figures 3(a) and 3(b). One can easily see the influence of the thermal diffusivities discontinuity on the solution of (5.7) and the importance of boundary conditions in the computations of the solutions of (5.20). The energies linked to the computed auxiliary functions have allowed the evaluation of macroscopic coefficients given in Table 1 ( $I_{2}$ denotes the "identity" matrix of order 2 ). 

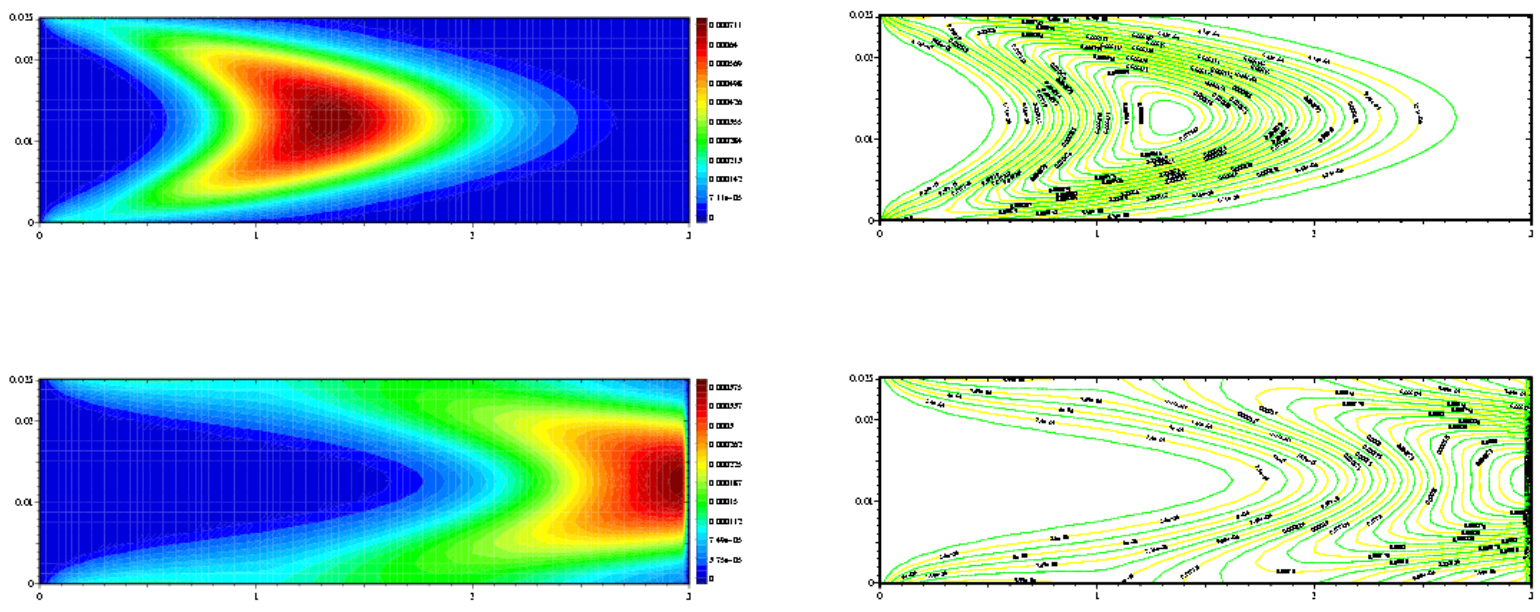

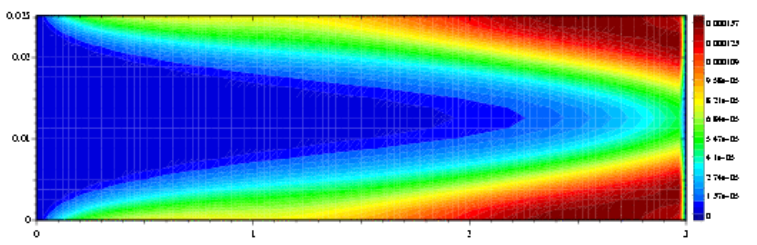

(a)

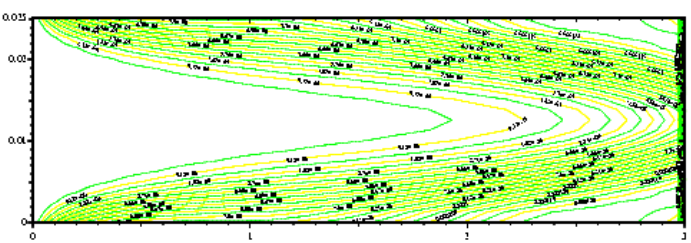

(b)

Figure 2. 2D-visualization of a flow in a capillary tube: $P e=2000, \Delta \theta=30 \mathrm{~K}$.

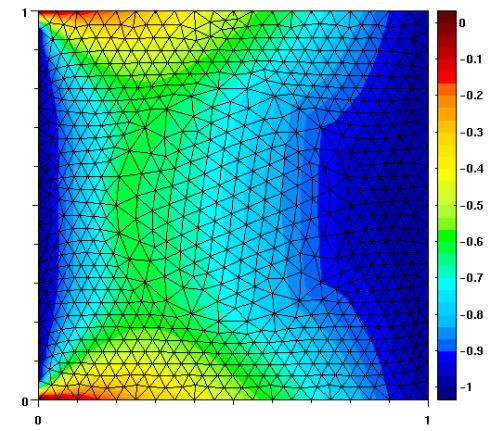

(a) $\omega_{1}, 1^{s t}$ cell, 1220 elements

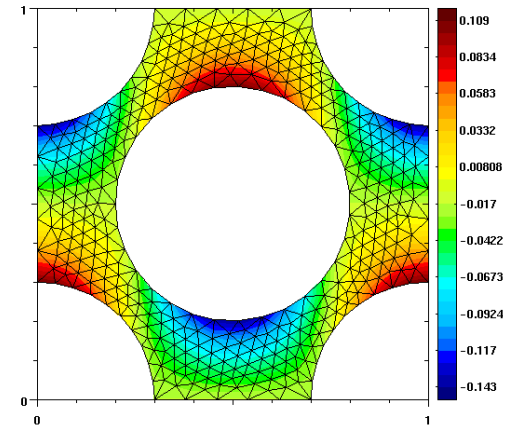

(b) $\omega_{2}, 2^{\text {nd }}$ cell, 409 elements

FiguRE 3. Auxiliary problem solutions (5.7) and (5.20).

Other computations must be performed with more realistic and thus more complex geometries. Nonsymetric cells or anisotropic media should improve the effect of tortuosity on thermodiffusion phenomena.

Acknowledgements. This work has been granted by the French petroleum society Total Fina Elf in the frame of research contract Elf EP $\mathrm{n}^{\circ}$ 11733. This scientific agreement between the Pau University and the society Total Fina Elf has allowed the financing of the thesis of B. Lacabanne. The authors wish here to thank this society for its support. 


\section{REFERENCES}

[1] G. Allaire, Homogenization and two-scale convergence. SIAM J. Math. Anal. 23 (1992) 1482-1518.

[2] G. Allaire, Homogénéisation des équations de Stokes et de Navier-Stokes. Thèse de doctorat de l'Université Paris VI (1989).

[3] G. Allaire, A. Damlamian and U. Hornung, Two-scale convergence on periodic surfaces and applications, in Proceedings of the International Conference on Mathematical of Flow through Porous Media, A. bourgeat et al. Eds., World scientific Pub., Singapore (1995).

[4] S.N. Antontsev and A.V. Domansky, Uniqueness generalizated solutions of degenerate problem two phase filtration. Numerical methods mechanics in continuum medium, Collection Sciences Research, Sbornik 15 (1984) 15-28 (in Russian).

[5] S.N. Antontsev, A.M. Meirmanov and V.V. Yurinsky, Homogenization of Stokes-Type equations with variable viscosity. Siberian Adv. Math. 8 Allerton Press, New York (1998).

[6] P. Bia and M. Combarnous, Les méthodes thermiques de production des hydrocarbures. Chap. 1: Transfert de chaleur et de masse. Revue de l'Institut Français du Pétrole, mai-juin (1975) 359-395.

[7] H. Brezis, Analyse fonctionnelle, théorie et applications. Masson, Paris (1983).

[8] R. Dautray and J.L. Lions, Analyse mathématique et calcul numérique pour les sciences et les techniques. 9 Vols., Masson, Paris (1985).

[9] J.I. Díaz, Two problems in homogenization of porous media. Preprint, Université Complutense de Madrid, MA-UCM 1999-25, (1999).

[10] J.I. Díaz and G. Galiano, Existence and uniqueness of solutions of the Boussinesq system with nonlinear thermal diffusion. Topological Methods in Nonlinear Analysis, Journal of the Juliusz Schauder Center 11 (1998) 59-82.

[11] J.I. Díaz, G. Galiano and A. Jungel, On a quasilinear degenerated system arising in semiconductors theory. Part I: existence and uniqueness of solutions. Centrum voor Wiskunde en Informatica, Modelling, Analysis and Simulation. Report MAS-R9723, (1997).

[12] G. Duvaut and J.L. Lions, Les inéquations en mécanique et en physique, Dunod, Paris (1972).

[13] G. Gagneux, Sur l'analyse de modèles de la filtration diphasique en milieu poreux, in Equations aux dérivées partielles et applications. Articles dédiés à J.L. Lions. Gauthier-Villars, Paris (1998) 527-540.

[14] G. Gagneux and M. Madaune-Tort, Analyse mathématique de modèles non linéaires de l'ingénierie pétrolière. Collection Mathématiques et Applications, Springer-Verlag, Heidelberg 22 (1996).

[15] U. Hornung, Homogenization and porous media. Interdisciplinary Appl. Math., No. 6, Springer, New York (1997).

[16] U. Hornung and W. Jäger, Diffusion, convection, adsorption, and reaction of chemicals in porous media. J. Differential Equations 92 (1991) 199-225.

[17] F. James, M. Sepulveda and P. Valentin, Modèle de thermodynamique statistique pour un isotherme d'équilibre diphasique multicomposant. Rapport de recherche du centre de mathématiques appliquées de l'École Polytechnique No. 223 (1990).

[18] J.L. Lions, Contrôle optimal de systèmes gouvernés par des équations aux dérivées partielles. Dunod, Paris (1968).

[19] J.L. Lions and E. Magenes, Problèmes aux limites non homogènes et applications. Vol. 1, Dunod, Paris (1968).

[20] F. Montel, Importance de la thermodiffusion en exploration et production pétrolières. Entropie No. 184/185 (1994) 86-93.

[21] R.A. Nicolä̈es, Existence, uniqueness and approximation for generalized saddle point problems. SIAM J. Numer. Anal. 19 (1982) 349-357.

[22] P.-A. Raviart and J.-M. Thomas, A mixed finite element method for second order elliptic problems, in Mathematical aspects of the finite element method, Lect. Notes Math. 606, Springer, Berlin (1977) 292-315.

[23] J.E. Roberts and J.-M. Thomas, Mixed and hybrid methods. Handbook of numerical analysis, finite element methods, Vol. II, finite element methods (Part. 1), Ciarlet P-G., Lions J-L. Eds. North Holland, Amsterdam (1991) 523-639 .

[24] E. Sanchez-Palencia, Non-homogeneous media and vibration theory. Springer-Verlag, Berlin (1980).

[25] D. Serre, Systèmes de lois de conservation. Tome 1, Fondations. Diderot Éditeur, Arts et Sciences, Paris (1996) 66-68.

[26] J-M. Thomas and D. Trujillo, Mixed finite volume methods, Intermat. J. Numer. Methods Engrg. 46 (1999) $1351-1366$.

To access this journal online:

www.edpsciences.org 